\title{
Methamphetamine Augments HIV-1 gp120 Inhibition of Synaptic Transmission and Plasticity in Rat Hippocampal Slices: Implications for Methamphetamine Exacerbation of HIV-Associated Neurocognitive Disorders.
}

\section{Ya Zheng}

University of Nebraska Medical Center College of Medicine

\section{Benjamin Reiner}

University of Nebraska Medical Center College of Medicine

Jianuo Liu

University of Nebraska Medical Center College of Medicine

Linda Xu

University of Nebraska Medical Center College of Medicine

Huangui Xiong ( $\nabla$ hxiong@unmc.edu )

University of Nebraska Medical Center https://orcid.org/0000-0003-3450-8876

\section{Research}

Keywords: Methamphetamine, HIV-1, synaptic transmission, long-term potentiation, microglia, inflammasome, hippocampus.

Posted Date: October 12th, 2021

DOl: https://doi.org/10.21203/rs.3.rs-955143/v1

License: (c) (i) This work is licensed under a Creative Commons Attribution 4.0 International License. Read Full License

Version of Record: A version of this preprint was published at Neurobiology of Disease on March 1st, 2022. See the published version at https://doi.org/10.1016/j.nbd.2022.105712. 


\section{Abstract}

Background: Methamphetamine (Meth) abuse and human immunodeficiency virus type 1(HIV-1) infection are two major public health problems worldwide. Being frequently comorbid with HIV-1 infection, Meth abuse exacerbates neurocognitive impairment in HIV-1-infected individuals even in the era of combined antiretroviral therapy. While a large body of research have studied the individual effects of Meth and HIV-1 envelope glycoprotein 120 (gp120) in the brain, far less has focused on their synergistic influence. To uncover the underlying mechanisms for increased incidence and severity of HIV-1-associate neurocognitive disorders (HAND) in HIV-1-infected patients with Meth abuse, we investigated acute individual and combined effects of Meth and gp120 on synaptic transmission and plasticity in CA1 region of rat hippocampus, a brain region known to be vulnerable to HIV-1 infection.

Methods: Experiments were conducted on the hippocampal slices prepared from 15-30 d-old male Spraque-Dawley rats. Field excitatory postsynaptic potentials were generated by electric stimulation of Schaffer collateral fibers and recorded in the CA1 region. Long-term potentiation (LTP) was induced by high frequency stimulation (HFS) or theta-burst stimulation (TBS). The individual and combined effects of acutely applied methamphetamine and HIV-1 gp120 synaptic physiology and underlying mechanisms were examined via electrophysiology, western blotting and immunohistochemistry. One-way and two-way ANOVA, followed by Tukey's multiple comparisons tests were employed for statistical analyses.

Results: Acute and localized application of Meth and gp120 each alone reduced short-term dynamics of input-output responses and frequency facilitation, and LTP induced by either HFS or TBS. A synergistic augmentation on activity-dependent synaptic plasticity was observed when Meth and gp120 were applied in combination. Paired-pulse facilitation results exhibited an altered facilitation ratio, suggesting a presynaptic site of action. Further studies revealed an involvement of microglia NLRP3 inflammasome activation in Meth augmentation of gp120-mediated attenuation of LTP.

Conclusions: Our results demonstrated Meth augmented gp120 attenuation of LTP in the hippocampus via microglial inflammasome activation. Since LTP is the accepted experimental analog of learning at the synaptic level, such augmentation may underlie Meth exacerbation of HAND observed clinically.

\section{Background}

Human immunodeficiency virus type 1 (HIV-1) brain infection often provokes cognitive and behavior deficits collectively termed as HIV-1-associated neurocognitive disorders (HAND) [1, 2] which was characterized by severe cognitive dysfunction and mortality in the pre-antiretroviral era $[1,3]$. The implementation of combined antiretroviral therapy (CART) has led to a sharp decline in the mortality attributed to AIDS and dramatically reduced incidence of severe form of HAND in infected individuals [2, 4]. The widespread of cART has also transformed the HIV-1 infection from a life-threatening illness to a manageable chronic disease [4]. Nevertheless, in the cART era, viral persistence in brain in a latent or restrict manner continues to play a significant disease-inciting role. Whilst the most severe form of HAND 
is now rare, milder forms of cognitive impairment persist amongst people living with HIV-1 and prevalence of these forms of cognitive impairment is in fact on the rising $[2,4]$. Although the underlying mechanisms remain obscure, several risk factors have been identified including, but are not limited to, HIV-1 envelope glycoprotein 120 (gp120)-induced neurotoxicity [5, 6] and comorbid drug abuse, in particular, the psychostimulant methamphetamine (Meth) $[7,8]$.

The viral protein gp120 has been identified as a potent neurotoxin involved in the HAND pathogenesis with deleterious effects at concentrations ranging from picromolar to nanomolar in vitro [9, 10], or higher [11]. Such potency might be reflexive of its biological relevance in vivo [12]. Indeed, soluble gp120 at high concentrations has been detected in the peripheral blood and secondary lymphoid organs of individuals with chronic HIV infection [13], in addition to anti-gp120 antibodies in cerebral spinal fluid of patients with HAND [14]. Studies have shown that gp120, shed off from HIV-1 virions and/or released from infected brain cells $[2,15]$, on the one hand, induces neuronal apoptosis, synaptic and dendrite dysfunction[16, 17]; and, on the other hand, causes immune activation and resultant production of neurotoxic molecules leading to the development of HAND [15]. The neurocognitive impairment and pathophysiological alterations observed in HIV-1-infected individuals can be attributed to direct and indirect effects of gp120 and are frequently associated with the use of psychostimulant drugs such as Meth [18, 19].

Meth is one of the most common abused drugs among individuals infected with HIV-1 [20]. Ample evidence indicates that Meth abuse not only increases the risk of HIV-1 infection [21, 22], but also exacerbates the cognitive deficits and neurodegenerative abnormalities in HIV-1-infected patients as well as in gp120 transgenic (gp120-tg) animals [23-26]. It has also shown that HIV-1 infection and Meth abuse each alone may cause neuronal injury and cognitive impairment $[27,28]$. In contrast, they in combination appear to produce more serious neurocognitive impairment than each alone. Indeed, Meth was found to exacerbate neurocognitive deficits in HIV-1-infected individuals and gp120-tg animals [29, 30]. However, the mechanisms underlying Meth exacerbation of HIV/gp120-associated cognitive impairment are incompletely understood.

The hippocampus is one of the most thoroughly investigated brain structure widely believed to be involved in learning and memory [31,32]. Increasing evidences indicate that impairment of hippocampal structure and function occurs in HIV-1-infected individuals and Meth abusers, which is associated with cognitive deficits [33-35]. To understand how Meth exacerbates HIV-1-associated neurocognitive impairment, we studied the acute effects of Meth and gp120 on synaptic transmission and plasticity in rat hippocampal brain slices. Our results showed that Meth and gp120 each alone had an inhibitory effect on short- and long-term synaptic plasticity in the CA1 region, and this inhibition was augmented when Meth and gp120 tested in combination. Further studies revealed a presynaptic site of action and involvement of microglia inflammasome activation in Meth- and gp120-mediated inhibition of synaptic activity.

\section{Materials And Methods}




\section{Materials}

Full-length HIV-1 ${ }_{\text {MN }}$ gp120 (gp120, Clade B) was purchased from Immunodiagnostics, Inc. (Woburn, MA). Aliquots of gp 120 were kept as $100 \mathrm{nM}$ stock solution at $-80^{\circ} \mathrm{C}$. The stock solution was diluted to desired concentrations with artificial cerebrospinal fluid (ACSF) 2-5 min before test. Meth was purchased from Sigma (St. Louis, MO, Cat \# M-8750) with DEA license \# RX0374974. All other chemicals, unless otherwise specified, were also from Sigma.

\section{Animals}

Male Sprague-Dawley rats (15- to 30-day-old) used for this study were purchased from Charles River Laboratories (Wilmington, MA). Animals were housed at constant temperature $\left(22^{\circ} \mathrm{C}\right)$ and relative humidity (50\%) under a regular light-dark cycle (light on at 7am and off at $5 \mathrm{pm}$ ) with free access to food and water. All animal use procedures were strictly reviewed by the Institutional Animal Care and Use Committee (IACUC) of University of Nebraska Medical Center (IACUC No. 19-085-07-FC).

\section{Hippocampal slices}

Rat hippocampal brain slices were prepared as previously described [36]. Briefly, animals were anesthetized with isoflurane, decapitated, and their brains were quickly removed from the cranial cavity. The brains were placed into an ice-cold $\left(4^{\circ} \mathrm{C}\right)$ oxygenated artificial cerebrospinal fluid (ACSF) environment. The hippocampi were dissected free and transverse hippocampal slices $(400 \mathrm{~mm}$ in thickness) were cut using a tissue chopper. The slices were kept in a humidified/oxygenated interface chamber at room temperature $\left(22^{\circ} \mathrm{C}\right)$ for at least $1 \mathrm{hr}$ before being transferred into a recording chamber. In the recording chamber, a single hippocampal slice, rested on a nylon mesh ring sat closely to the bottom of the chamber fully, was submerged in a continuously perfused ACSF solution at a constant flow rate of $2 \mathrm{ml} / \mathrm{min}$ with the use of a peristaltic pump (Rainin Instrument Co., Woburn, MA). The ACSF contained (in mM): $\mathrm{NaCl}$ (124.0), $\mathrm{KCl}$ (3.0), $\mathrm{CaCl}_{2}$ (2.0), $\mathrm{MgCl}_{2}$ (2.0), $\mathrm{NaH}_{2} \mathrm{PO}_{4}$ (1.25), $\mathrm{NaHCO}_{3}$ (26.0) and Glucose (10.0), equilibrated with $95 \% \mathrm{O}_{2}$ and $5 \% \mathrm{CO}_{2}$, had a pH of 7.4-7.5 and an osmolarity of $\sim 305 \mathrm{mOsm}$. The temperature of the perfusion was maintained at $30 \pm 1^{\circ} \mathrm{C}$ with an automatic temperature controller (Warner Instrument Corp., Hamden, CT). Biological reagents were applied onto the slices and incubated in humidified/oxygenated interface chamber for 40-60 min prior to conducting electrophysiology recordings.

\section{Electrophysiology}

Field excitatory post-synaptic potentials (fEPSPs) were generated by test pulses applied every 20s (low frequency) with a constant current (100-300 mA, 40 ms in duration) stimulation of Schaffer collateralcommissural axons using an insulated bipolar tungsten electrode. The stimulation intensity was adjusted to generate approximately $40-50 \%$ of a maximal response. The evoked fEPSPs were recorded with an Axopatch-1D amplifier (Molecular Devices, San Jose, CA) in the CA1 dendrite field and Clampex 10 (Molecular Devices) was used for data acquisition. The recording microelectrodes were made from 
borosilicate glass capillaries (WPI, Sarasota, FL) with inner filaments that enable quick back-filling with ACSF (resistance of 1-5 MW). Electrical signals were filtered at $1.0 \mathrm{kHz}$ and digitized at $5.0 \mathrm{kHz}$ using a Digidata 1440 interface (Molecular Devices). Data were stored on a desktop PC and analyzed off-line using pCLAMP 10 software (Molecular Devices) and OriginPro 2019b (OriginLab, Northampton, MA). To eliminate the possibility that gp120 and Meth activated inhibitory interneurons, a portion of experiments were conducted in the presence of $20 \mathrm{mM}$ picrotoxin, except those testing the effects of Meth/gp120 on basal synaptic transmission. To prevent epileptiform activities, a surgical cut was made between CA1 and $\mathrm{CA} 3$ areas after the slice had been transferred to the recording chamber.

For LTP experiment a 30 min-baseline recording was conducted once the adjustment of stimulation parameters was achieved. High frequency stimulation (HFS, $100 \mathrm{~Hz}, 500 \mathrm{~ms} /$ train ' 3) was delivered 30 min after the start of recording on each slice with an inter-train interval of $20 \mathrm{~s}$. Each recording trial was an average of 3 consecutive sweeps. The initial slope of fEPSP was analyzed and expressed in percentage of basal level, i.e., the average of initial slopes from the first $30 \mathrm{~min}$ in HFS and $15 \mathrm{~min}$ in theta-burst stimulation (TBS) were treated as $100 \%$, respectively. In bar graphs, the magnitudes of LTP were quantified as the average of entire 60 min recordings after HFS and entire 45 min recordings after TBS. All data were expressed as the mean \pm standard error of the mean (SEM) unless otherwise indicated. To mimic physiological condition, the TBS was also employed to induce LTP (TBS-LTP). The TBS protocol, as described previously [37], was composed of 5 trains of 4 pulses (100 Hz) at a rate of 1 train per $200 \mathrm{~ms}$ and repeated twice with an interval of $10 \mathrm{~s}$. Like in HFS-LTP, a baseline recording was conducted for 15 min before TBS stimulation. Changes in LTP were evaluated with the same method as HFS-LTP.

Input-output (I-O) tests were conducted by setting the stimulus intensity to evoke $40-50 \%$ of maximal fEPSPs. The stimulus intensity was set an increment of $0.1 \mathrm{~mA}$ from $0.2 \mathrm{~mA}$ to $0.8 \mathrm{~mA}$ and resultant fEPSPs were recorded and analyzed. PPF curves were generated by testing the Schaffer-collateral pathway with twin pulses at a fixed pulse duration of $40 \mathrm{~ms}$ with varying inter-pulse interval (IPI) at a 20 $\mathrm{ms}$ increment from $20 \mathrm{~ms}$ to $100 \mathrm{~ms}$. The paired pulses were delivered at 20s intervals and 3 consecutive responses were averaged at each PPF test. The degree of facilitation was determined as the increase in ratio of the amplitude (or initial slope) of the second response over the first response in each pair. Since there was no significant difference between measurement in amplitude and initial slope, the data were pooled.

Frequency-dependent synaptic plasticity tests were performed at half-maximal fEPSP on each slice. After 20 minutes of slice acclimation, a ten-pulse stimulus train at $1.0,5.0$ or $10.0 \mathrm{~Hz}$ was applied to the Schaffer collaterals and the resultant responses were recorded. The slices were allowed to recover over 20 minutes before a different stimulation burst was given and recorded. The 10 pulse- burst applied each time was at decreased inter-pulse intervals from $1.0 \mathrm{~s}$ to $0.2 \mathrm{~s}$ and then to $0.1 \mathrm{~s}$, respectively. The initial slope from each pulse (2-10 ${ }^{\text {th }}$ pulses) in the recorded burst was analyzed and expressed as the percentage of the first pulse (taking the first pulse as 100\%). The recordings from the same experimental group at the same time point were averaged and graphed. 


\section{Western blot analysis}

Following five hours' treatment of reagents, rat hippocampal slices were harvested for Western blot analysis. The lysates of brain tissue were prepared using RIPA lysis buffer (Thermo Fisher Scientific, Waltham, MA) supplemented with a protease inhibitor cocktail (Thermo Fisher Scientific). After quantification of protein concentrations with BCA protein assay kit (Thermo Fisher Scientific), $15 \mu \mathrm{g}$ of total protein was loaded onto $8-12 \%$ SDS-polyacrylamide gels, followed by the protocol as previously described $[38,39]$. The primary antibodies used were BCL2-associated X protein (Bax; 1:500; Cell Signaling Technology), B cell lymphoma 2 (Bcl-2;1:1000; Cell Signaling Technology), caspase-1 (1:500; Santa Cruz), Iba-1 (1:500; FUJIFILM Wako), IL-1 $\beta$ (1:2500; Abcam), NLRP3 (1:500; Novusbio), ASC (1:500; Santa Cruz), caspase-8 (cas8; 1:500, Cell Signaling Technology), GAPDH (1:20000; Sigma), and $\beta$-actin (1:5000; Sigma). The immunoblots were detected by an enhanced chemiluminescence (ECL) system (Thermo Fisher Scientific) and imaged by the FluorChem M system (ProteinSimple, Santa Clara, CA). Band densities of labeled proteins were measured by ImageJ software ( $\mathrm{NIH}$, Bethesda, MD).

\section{Immunofluorescence Staining}

After $4 \mathrm{hr}$ treatment with Meth and/or gp120, rat hippocampal slices were collected into $4 \%$ freshly made PFA (dissolved in $0.01 \mathrm{M} \mathrm{PBS}$ ) overnight at $4^{\circ} \mathrm{C}$. Next day, the slices were moved into $10 \%$ sucrose for fixation until sinking to the bottom of EP tubes, followed by $20 \%$ and $30 \%$ sucrose in sequence in the same way. Then the optimum cutting temperature (O.C.T.) compound (Tissue-Tek ${ }^{\circledR} 4583$, Sakura) was used to embed the slices on dry ice. Coronal sections of hippocampal embedded mass were cut at 10-20 $\mu \mathrm{m}$ in thickness using a freezing microtome (CM1850, Leica). After post-fixation in 4\% PFA for 30 min, sections were washed with $0.01 \mathrm{M}$ PBS three times, permeabilized in $0.01 \mathrm{M}$ PBS containing $0.02 \%$ Triton $\mathrm{X}-100$ (PBST) for $20 \mathrm{~min}$, and then blocked in PBST containing 10\% goat serum for $30 \mathrm{~min}$ at room temperature. Sections were then incubated in a humidified chamber overnight at $4^{\circ} \mathrm{C}$ with the primary antibody (anti-CD11b: 1:200, AbD MCA275G; anti-lba-1: 1:200, Wako 019-19741; anti-ASC: 1:50, sc514414) in PBST with 10\% goat serum. Next day, the sections were washed three times with PBST, followed by incubation $1 \mathrm{~h}$ with the secondary antibody (goat anti-mouse IgG H\&L (Alexa Fluor ${ }^{\circledR} 488$ ), 1:1000, ab150077; goat anti-mouse IgG H\&L (Alexa Fluor® 594), 1:1000, A-11032; goat anti-rabbit IgG H\&L (Alexa Fluor ${ }^{\circledR} 488$ ), 1:1000, A-11034) in PBST solution at room temperature. Then 4',6-diamidino-2phenylindole (DAPl; 1:10000, Invitrogen ${ }^{\text {TM }}$ D1306) in 0.01 M PBS was used to stain nuclei for 10 min. Finally, each slide was aspirated with the reagents and covered by a coverslip under the Fluoroshield Mounting Medium (P36934; Thermo Fisher Scientific). Images were acquired from the region of dentate gyrus by a confocal microscope (LSM 710; Zeiss). 10 regions of hippocampal slides in 4 rats were analyzed for each group, and quantified by ImageJ software (NIH, Bethesda, MD).

\section{Statistical Analyses}

Statistical analyses were performed using GraphPad® Prism 9.0 (GraphPad Software, San Diego, CA). All data were graphed, and analyzed using one-way or two-way ANOVA, followed by Tukey's 
multiple comparisons test to determine significant group differences. Values were expressed as mean \pm SEM and $p<0.05$ was considered statistically significant.

\section{Results}

\section{Meth and gp120 inhibition of synaptic transmission in rat hippocampal slices}

While the combined effects of Meth exposure and gp120 on synaptic function were examined in gp120 transgenic (gp120-tg) rodents, few studies have investigated their acute effects in the central nervous system (CNS). To explore their acute effects on synaptic transmission in the CNS, we examined inputoutput (I-O) responses by electrical stimulation of Shafer-collateral fibers and recording of resultant fEPSPs in the CA1 dendritic field, using a protocol with a fixed IPI and variable stimulus current intensities from $0.2 \mathrm{~mA}$ to $0.8 \mathrm{~mA}$ in a $0.2 \mathrm{~mA}$ increment. Application of Meth and gp120 each alone onto the CA1 region of hippocampal slices significantly reduced average initial slopes at all stimulus intensities tested when compared with the average initial slope in control group (Figure 1). The average initial slope was further reduced when Meth and gp120 were administered in combination (Figure 1). The reduction was statistically significant when compared with either Meth or gp120 alone group, demonstrating synergistic effects of Meth and gp120 inhibition of synaptic transmission.

\section{Meth augmentation of HIV-1 gp120 inhibition of LTP}

Neurocognitive deficits tend to be worse in HIV-1-infected individuals with Meth abuse and the underlying mechanisms remain obscure. To explore the potential mechanisms for Meth exacerbation of neurocognitive deficits seen in HIV-1-infected individuals, we studied the effects of Meth and gp120 each alone and in combination on HFS-induced LTP in the CA3-CA1 synapses in rat hippocampal slices because memories are believed to be encoded by modification of synaptic strength and LTP is widely considered as one of the major cellular mechanisms that underlies learning and memory. As shown in Figure 2B, employment of the HFS protocol produced a robust LTP in untreated slices (control). The average magnitude of LTP was $237.68 \pm 9.88 \%$ of baseline (Figure $2 C$, Mean $\pm S E M, n=23$ ). Application of Meth and gp120 individually reduced LTP magnitudes to $156.35 \pm 7.64 \%(n=22)$ and $171.48 \pm 6.89 \%(n=20)$, respectively. The difference for each test was statistically significant compared to control $\left(P_{\text {Meth }}<0.05, P_{\mathrm{gp} 120}<0.001\right)$. Application of Meth and gp120 in combination produced further significant reduction on LTP magnitude to $123.48 \pm 4.09 \%(p<0.05, \mathrm{n}=20)$, demonstrating Meth potentiation of gp120-induced inhibition of LTP. To mimic the stimulation under physiological condition, we employed widely used theta-burst stimulation (TBS) to induce LTP and tested effects of Meth and gp120 on TBS-induced LTP. Likewise, TBS also produced a robust LTP in the hippocampal slices (Figure 2D). Meth and gp120 attenuated the average LTP magnitudes to $150.58 \pm 28.14 \%(n=6)$ and $156.09 \pm 17.02 \%(n=6)$ of baseline, respectively (Figure $2 E)$. In comparison to the LTP magnitude $273.94 \pm$ $13.96 \%$ of baseline in control $(n=5)$, the differences were statistically significant $(p<0.001)$. Application of Meth and gp120 in combination further reduced LTP magnitude to $123.19 \pm 8.46 \%$ of baseline $(n=7)$. Compared with the LTP magnitudes when Meth and gp120 were applied alone, the difference was 
statistically significant between Meth+gp120 and gp120 groups $(p<0.05)$, but not between Meth+gp120 and Meth groups $(p>0.05)$, demonstrating a synergic effect of Meth and gp120 on LTP in the hippocampal slices.

\section{Effects of Meth and gp120 on frequency-dependent synaptic responses}

In monosynaptic transmission, the magnitude of the postsynaptic responses evoked by pre-synaptic stimulation is intrinsically dependent on stimulation frequency [40]. To examine whether Meth and gp120 alter frequency-dependent short-term synaptic plasticity in CA3-CA1 synapses, we recorded the fEPSPs elicited by a stimulation paradigm of ten-pulse train at the frequency of 1,5 and $10 \mathrm{~Hz}$, respectively (Figure 3A). The responses evoked by the consecutive pulses in the train were quantified as the percent change of each of the nine consecutive fEPSPs with respect to the first fEPSP. Meth and gp120, applied alone or in combination, reduced the frequency-associated facilitation of synaptic response as reflected by the decrease of fEPSP slope at $5 \mathrm{~Hz}$ and $10 \mathrm{~Hz}$ (Figures 3B-D). The reduction of frequency-associated facilitation of synaptic response was statistically significant compared to the control $\left(P_{\text {Meth, Meth+gp } 120<}\right.$ $\left.0.001, P_{\mathrm{gp} 120}<0.05\right)$, illustrating Meth and gp120 reduction of frequency-dependent synaptic response in the hippocampal brain slices. When applied in combination, Meth and gp120 were found to further reduce frequency facilitation of synaptic responses when compared to Meth alone at the frequency of 5 $\mathrm{Hz}(p<0.01)$ or gp120 alone at $10 \mathrm{~Hz}$ (Figure 3E, $p<0.05)$.

\section{Meth- and gp120-alteration of paired-pulse facilitation ratio}

To understand the site of action of Meth and HIV-1 gp120 on synaptic transmission, we measured the paired-pulse ratio (PPR), the ratio of the fEPSP initial slope (or amplitude) of the second response to that of the first. Paired-pulse stimulation with different inter-pulse intervals (IPIs) produced robust responses in control slices as shown in Figure 4A. Meth significantly increased PPR with stimulation IPIs of 40,60 and $100 \mathrm{~ms}$ (two-way ANOVA with Tukey's post hoc test, $P_{40}<0.01, P_{60,100}<0.05$ ) (Figures 4A-C), while gp120 produced significant increase $(P<0.05)$ of PPR only in the IPI of $40 \mathrm{~ms}$ when compared to the control group. These results suggested a presynaptic site of action for Meth and gp120. The PPR was further increased when Meth and gp120 were applied in combination, illustrating an augmented effect of Meth and gp120 on the PPR.

\section{Activation of microglia in the hippocampus by combined effect of Meth and gp120}

Ample evidence indicates that microglia activation and resultant proinflammatory responses alter neuronal and synaptic function in the brain. To determine potential involvement of microglia activation in Meth- and gp120-associated inhibition of synaptic plasticity, we examined whether acute application of Meth and gp120 in the CA1 region produced microglia activation and resultant production of IL-1 $\beta$ in rat hippocampal slice. As shown in Figures 5A-D, Meth and gp120 each alone induced microglia activation evidenced by an increase of CD11b/DAPI double positive cells in the hippocampus when compared to the control group ( $P<0.01$ vs. Meth; $P<0.05$ vs. gp120). Most notably, remarkable increase in microglia activation was observed when Meth and gp120 were applied in combination $(P<0.001)$. The observed 
increase of microglia activation was in consistent with immunostaining, western blot results that the expression of Iba-1 protein, a microglia marker, and prolL-1 $\beta$, a product of inflammatory response, were significantly upregulated by Meth and gp120 applied in combination (Figures 5E-F). To explore the potential involvement of inflammasome in Meth- and gp120-mediated microglial activation, a highly selective and irreversible caspase-1 inhibitor Ac-YVAD-CMK $(10 \mu \mathrm{M})$ [41] was applied onto the hippocampus slices $1 \mathrm{hr}$ prior to the application of Meth+gp120. Under this experimental condition, microglial activation was markedly suppressed as evidenced by reduced expression levels of pro-IL-1 $\beta$ and Iba-1 (Figures 5E-F, $P_{\mathrm{lba}-1}<0.001, P_{\mathrm{IL}-1 \beta}<0.01$ ), suggesting the involvement of inflammasome in Meth- and gp120-associated microglial activation.

\section{Involvement of NLRP3 inflammasome in microglial activation induced by combined treatment of Meth and gp120}

After observation on caspase-1 inhibitor Ac-YVAD-CMK suppression of microglia activation, we further analyzed the expression levels of inflammasome composition proteins by western blot and examined the adaptor protein ASC oligomerization in microglia using immunofluorescent staining. As a critical component of the inflammasome, ASC protein was found to aggregate, appearing as "speck"-like spots located at the nuclear periphery in microglia treated with Meth and gp120 (Figure 6A). Such an aggregated ASC was only observed after treatment with Meth and gp120 in combination, but not in each treated alone, indicating the aggregation of inflammasome platform following treatment of Meth and gp120. To further examine the protein expression levels of inflammasome components, we also conduct western blot assay on hippocampal slices and exemplary gels were exhibited in Figure 6B. As shown in Figures 6D-E, upregulated levels of ASC protein and procaspase- 1 were evident in Meth+gp120 group compared to the gp120-treated group $(p<0.05)$, and Meth-treated group $(p<0.05)$, respectively. However, there was no significant difference in NLRP3 expression levels between the individual and combined application of Meth and gp120 (Fig. 6C, $P>0.05$ ). These results demonstrated that application of Meth and gp120 in combination gave rise to inflammasome activation via ASC oligomerization and caspase-1 activation.

\section{Discussion}

Meth abuse and HIV-1 infection are two major public health issues worldwide. Being frequently comorbid with HIV infection, Meth abuse exacerbates neurocognitive deficits in HIV-1-infected individuals even in the era of cART $[6,19,30]$. While many studies have investigated the individual effects of Meth and gp120 in the brain [33,42], far less has been focused on their synergistic influence. In the present study, we examined acute effects of Meth and gp120, when applied individually or in combination, on short- and long-term synaptic plasticity in the CA1 region of rat hippocampal slices. Our results demonstrated that: (i) Meth and gp120 each alone attenuated short- and long-term synaptic plasticity as reflected by a decrease of $\mathrm{I} / \mathrm{O}$ responses, a decline of fEPSP slope percentage in frequency tests, and a reduction on the

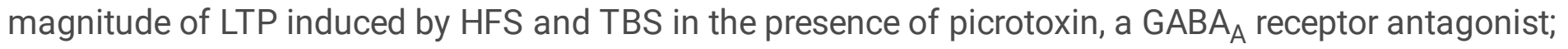

(ii) Meth augmented gp120-assicated attenuation of short- and long-term synaptic plasticity when 
applied in combination; (iii) Meth and gp120 increased paired-pulse facilitation ratio, suggesting a presynaptic site of action; (iv) Meth and gp120 each alone or in combination induced significant microglia NLRP3 inflammasome activation in the hippocampus as revealed by increased expression levels of prolL-1 $\beta$, NLRP3, ASC and procaspse-1; (v) application of Ac-YVAD-CMK, a potent and irreversible inhibitor of the inflammatory enzyme caspase-1 [41] blocked Meth+gp120-mediated attenuation of LTP, indicating an involvement of NLRP3 inflammasome activation in Meth- and gp120associated attenuation of short- and long-term synaptic plasticity in the hippocampus.

The hippocampus has been a major brain system for studies of synaptic plasticity in the context of putative information-storage mechanisms in the brain. It is one of the most intensively investigated structure in the brain and activity-dependent synaptic plasticity is a prominent feature of hippocampal synapses. The most studied form of activity-dependent synaptic plasticity in the hippocampus is LTP, a widely accepted model linking synaptic plasticity with learning and memory $[43,44]$, although other forms of activity-dependent plasticity have also been found $[45,46]$. Ample evidence indicates that the hippocampus is the primary site of spatial learning and long-term memory formation $[47,48]$. Alteration of hippocampal LTP induction may lead to impairment of learning and memory [47]. To understand the mechanisms for Meth exacerbation of HAND seen in HIV-1-infected individuals with Meth abuse, we investigated acute effects of Meth and gp120 on synaptic transmission and plasticity in CA3 to CA1 synapses in the hippocampus, a brain region known to be vulnerable to HIV-1 infection [49]. Our results showed that localized application of Meth and gp120 each alone onto the CA1 region of the hippocampal slices reduced $\mathrm{I}-\mathrm{O}$ responses and frequency facilitation, increased paired-pulse facilitation ratio, and attenuated LTP induced by HFS as well as by TBS. A synergistic augmentation on inhibition of synaptic transmission and plasticity was observed when Meth and gp120 were applied in combination.

Alteration on paired-pulse facilitation ratio suggests presynaptic involvement in Meth- and gp120mediated effects. The reduction of I-O responses and frequency facilitation is indicative of dysfunction of synaptic transmission whereas the attenuation of LTP implies an impairment of learning and memory. The synergistic effects of Meth and gp120 revealed in this study may represent a potential mechanism, at least in part, for Meth-associated exacerbation of HAND observed clinically, even in the era of cART, in HIV-1-infected individuals with Meth dependence $[29,30,50]$.

Much of the neurotoxicity associated with HIV-1 brain infection has been attributed to the effects of viral envelope protein gp120 shed off from infected cells. It has been demonstrated that gp120 causes neural cell apoptosis [51] and oxidative stress [52], disrupts blood-brain barrier (BBB) [53] and induces neuroinflammation [54], ultimately leading to cognitive impairment and pathogenesis of HAND. In contrast to gp120, the neurotoxic effects of Meth, although well-studied, are not well-defined [55, 56]. While a plethora of research has investigated individual neurotoxic effects of gp120 and Meth in the brain, few has been done on their synergic influence on synaptic transmission and plasticity (LTP). In this study we observed that gp120, when applied alone, attenuated hippocampal LTP, which is consistent with the findings that gp120 inhibited short- and long-term potentiation in the hippocampus when applied acutely onto the hippocampal slices $[57,58]$, administered to the brain via intracerebroventricular infusion 
(for 3-5 days) [59], or overexpressed in the brain in gp120-transgenic animals [24,60]. We also observed that Meth reduced LTP when applied acutely, which is in agreement with the results that Meth reduced LTP recorded either in the CA1 region of mouse hippocampus in vitro [61] and rat hippocampus ex vivo $[62,63]$ or in the dentate gyrus in vivo in reinstated rats [64], along with the findings observed in the medial portion of the prefrontal cortex [65] and in the cortical-striatal region of rats with selfadministration of Meth [66]. In addition to its suppressive effect, Meth was also found, when administered for a long-term with a low dose, to enhance LTP in differentiating and mature mouse dentate gyrus neural cells [67]. Nevertheless, limited studies have been done on their combined effects on LTP in the hippocampus. Current literature search on PubMed displayed only one publication investigated the combined effect of Meth and gp120 on LTP in HIV gp120-tg animals and that study demonstrated Meth enhanced gp120 reduction on LTP and impaired learning and memory [24]. The results from that study can be utilized as a validation of acute effects of Meth and gp120 we observed in vitro in an animal model in vivo, solidifying Meth augmentation of gp120-associated attenuation of LTP in the hippocampus.

HFS and TBS are generally accepted protocols for LTP induction. While HFS is widely used in numerous experimental studies $[68,69]$, the TBS protocol has also gained broad acceptance in studying activitydependent synaptic plasticity because it is intended to mimic physiological theta-frequency activity in the brain [70-73] and has been found even more efficient than other forms of stimulation for LTP induction [74]. We used both HFS and TBS stimuli for LTP induction since the two types of stimuli were found to activate an experimental model leading to transformation from working memory to long-term memory $[75,76]$. Although Meth ang gp120 each alone decreased the magnitude of LTP induced by HFS and TBS, they appear to have stronger effects on TBS-induced LTP than HFS-induced LTP, indicating that Meth and gp120 interfere activity-dependent synaptic plasticity in a physiological condition in the hippocampus. As the hippocampal electroencephalogram (EEG) is dominated by a striking theta activities when animals are engaged in active motor behaviors such as investigatory activities [71, 77] and theta rhythm is considered a temporal correlate of memory storage processes in rat [77], the reduction on the magnitude of TBS-induced LTP by Meth and gp120 applied in combination may implicate the mechanism, at least in part, for HAND pathogenesis and Meth exacerbation of HAND.

Mounting evidence indicates that Meth abuse exacerbates HAND in HIV-1-infected individuals [30, 50]. Animal models of NeuroAIDS revealed that gp120 protein per se can cause cognitive impairment $[6,23$, 24]. Furthermore, a cross-species study demonstrated that HIV in humans and gp 120 in mice impaired learning and executive functions which were worsened by Meth abuse in both species [6]. Although exact mechanisms for Meth exacerbation of HIV/gp120-associated neurocognitive impairment remain ambiguous, various possibilities have been proposed including, but are not limited to, oxidative stress [78, 79], microglia activation and resultant neural injury [19, 38, 39], disruption of the blood-brain barrier [80, 81] and synaptodendritic dysfunction and damage in the adult cortex and hippocampus [24, 26]. Our results showed the synergic effect of Meth and gp120 on LTP was significantly attenuated by Ac-YVAD$\mathrm{CMK}$, a potent and irreversible inhibitor of the inflammatory enzyme caspase-1[41]. As the inflammasome consists of NLRP3 (receptor sensor), ASC (bridge protein) and caspase-1 $\beta$ (IL-1 converting enzyme), the 
Ac-YVAD-CMK-mediated attenuation of the synergic effect of Meth and gp120 on LTP suggests an involvement of NLRP3 inflammasome activation on Meth and gp120 inhibition of LTP. Further studies revealed that Meth and gp120 induced microglia activation (Figure. 5) and increased the expression levels of Iba- 1 and prolL-1 $\beta$. The prolL-1 $\beta$ can be cleaved into IL-1 $\beta$ by caspase- 1 and the elevated IL-1 $\beta$ may contribute to Meth and gp120-associated inhibition of LTP in the CA1 region of the hippocampus. This assumption is based on the fact that IL-1 $1 \beta$ receptors are present at high levels in the hippocampus [82] and hippocampal LTP was impaired via mechanisms associated with microglial activation and IL-1 $\beta$ activity $[83,84]$. Indeed, many studies have demonstrated that IL- $1 \beta$ inhibits LTP in the hippocampus [85-88]. In view of the results observed by others mentioned above and combined with our previous data that Meth induced inflammasome activation and increased production of IL-1 [39] and potentiated gp120-induced microglial activation in primary microglial cell cultures [38], we argue that Meth enhancement of gp120-associated microglial inflammasome activation may contribute to, at least in part, their inhibitory effects on synaptic transmission and plasticity in the hippocampus in vitro although further experiments are needed to test this argument.

It is worth pointing out that the concentrations of Meth and gp120 used in this study were physiologically relevant as determined in our previous study [38]. The rationale for using physiologically relevant doses is to mimic the disease condition of HAND, a chronic illness with low levels of viral replication in the era of cART. Although the actual in vivo plasma and brain tissue concentrations of gp120 in chronically HIVinfected patients remain unclear, the levels detected in the serum of HIV-infected individuals were 1-8 $\mathrm{nM}$ [9]. Given that $1 \mathrm{nM}$ of gp120 is $\sim 0.12 \mu \mathrm{g} / \mathrm{ml}$ [10], the soluble gp120 concentrations were estimated between 120 and $960 \mathrm{ng} / \mathrm{ml}[11]$. More higher levels of gp120 were also detected in secondary lymphoid organs of individuals with chronic viral infection ranging from $500 \mathrm{ng} / \mathrm{ml}$ to $5 \mu \mathrm{g} / \mathrm{ml}$ when all forms of gp120 were added, including soluble, cell- and virion-associated gp120 [11, 13]. The concentrations of gp120 used in this study were 100-500 pM which were below physiological concentrations detected by Oh et al. [9]. It is also worth noting that the concentrations of Meth used in this study were based on the biodistribution profiles from previous studies [89]. The Meth levels in the circulating blood of abusers were $2.0 \mu \mathrm{M}$ on an average, with the highest of $11.1 \mu \mathrm{M}$ [89]. The Meth levels in the brain of abusers can be further estimated according to the tissue-to-serum ratio of Meth in animals, with an average of 9.7 for brain distribution [90]. Based on this distribution ratio, the dose range of Meth was 6-150 $\mu \mathrm{M}$. To reflect the conditions in human subjects, the Meth concentrations $(20 \mu \mathrm{M})$ used in this study were within this range. Thus, the results obtained by using physiologically relevant concentrations of gp120 and Meth may have implications for HAND pathogenesis in the era of CART that lower levels of viral replication do not produce neuronal injury, but do cause microglial activation and resultant proinflammatory responses in the presence of Meth, leading to alteration of neuronal/synaptic functions such as reduction of LTP, a potential mechanism underlying Meth exacerbation of HAND seen in HIV-1-infected patients.

In summary, our results demonstrated that Meth and gp120 each alone, when applied acutely, had an inhibitory effect on short-term and long-term synaptic plasticity in the CA1 region of rat hippocampal slices as revealed by tests on I/O responses, frequency facilitation and LTP. Meth augmented gp120 inhibition of synaptic plasticity when applied in combination. Changes in paired-pulse facilitation suggest 
presynaptic involvement in Meth- and gp120-associated actions. The synergic effects of Meth and gp120 on LTP was blocked by Ac-YVAD-CMK, a potent inhibitor of the inflammatory enzyme caspase-1, suggesting an involvement of NLRP3 inflammasome activation in Meth augmentation of gp120 reduction of LTP in the hippocampus. The results obtained in the present study may implicate a potential mechanism, at least in part, for Meth-associated exacerbation of HAND observed in HIV-1-infected individuals with Meth dependence in the era of cART.

\section{Abbreviations}

ACSF: artificial cerebrospinal fluid; BBB: blood brain barrier; CART: combined antiretroviral therapy; CNS: central nervous system; fEPSP: field excitatory post-synaptic potentials; gp120: HIV-1 envelope glycoprotein 120; HAND: HIV-1-associated neurocognitive disorders; HFS: high frequency stimulation; HIV-1: human immunodeficiency virus type 1; IPI: inter-pulse interval; LTP: long-term potentiation; Meth: methamphetamine; NLRP3: NOD-, LRR- and pyrin domain-containing protein 3; PPR: paired-pulse ratio; TBS: theta-burst stimulation;

\section{Declarations}

\section{Author Contributions}

H.X. directed study; Y.Z., H.X analyzed, interpreted results and wrote the manuscript; Y.Z., B.R., J. L., L.X. performed experiments and collected/analyzed data; All authors read and approved the manuscript.

\section{Funding}

This work was supported by the grant R01DA050540 (H.X.) from National Institute on Drug Abuse, National Institutes of Health.

\section{Availability of data and materials}

The data that support the findings of this study are available from corresponding author upon reasonable request.

\section{Ethics approval and consent to participate}

All experimental protocols and animal care were carried out in accordance with the National Institutes of Health Guide for the Care of Laboratory Animals in Research and approved by the Institutional Animal Care and Use Committee of the University of Nebraska Medical Center (IACUC \# 19-085-07-FC).

\section{Consent for publication}

Not applicable

\section{Competing Interests}


The authors declare that they have no competing of interests.

\section{References}

1. Antinori A, Arendt G, Becker JT, Brew BJ, Byrd DA, Cherner M, Clifford DB, Cinque P, Epstein LG, Goodkin K, et al. Updated research nosology for HIV-associated neurocognitive disorders. Neurology. 2007;69:1789-99.

2. Saylor D, Dickens AM, Sacktor N, Haughey N, Slusher B, Pletnikov M, Mankowski JL, Brown A, Volsky DJ, McArthur JC. HIV-associated neurocognitive disorder - pathogenesis and prospects for treatment. Nat Rev Neurol. 2016;12:309.

3. Navia BA, Cho ES, Petito CK, Price RW. The AIDS dementia complex: II. Neuropathology. Ann Neurol. 1986;19:525-35.

4. Heaton RK, Franklin DR, Ellis RJ, McCutchan JA, Letendre SL, Leblanc S, Corkran SH, Duarte NA, Clifford DB, Woods SP, et al. HIV-associated neurocognitive disorders before and during the era of combination antiretroviral therapy: differences in rates, nature, and predictors. J Neurovirol. 2011;17:3-16.

5. Sanchez AB, Varano GP, de Rozieres CM, Maung R, Catalan IC, Dowling CC, Sejbuk NE, Hoefer MM, Kaul M. Antiretrovirals, Methamphetamine, and HIV-1 Envelope Protein gp120 Compromise Neuronal Energy Homeostasis in Association with Various Degrees of Synaptic and Neuritic Damage. Antimicrob Agents Chemother. 2015;60:168-79.

6. Kesby JP, Markou A, Semenova S. Cognitive deficits associated with combined HIV gp120 expression and chronic methamphetamine exposure in mice. European neuropsychopharmacology: the journal of the European College of Neuropsychopharmacology. 2015;25:141-50.

7. Lanman T, Letendre S, Ma Q, Bang A, Ellis R. CNS Neurotoxicity of Antiretrovirals. J Neuroimmune Pharmacol. 2021;16:130-43.

8. Sanchez AB, Kaul M. Neuronal Stress and Injury Caused by HIV-1, cART and Drug Abuse: Converging Contributions to HAND. Brain Sci 2017, 7.

9. Oh SK, Cruikshank WW, Raina J, Blanchard GC, Adler WH, Walker J, Kornfeld H: Identification of HIV-1 envelope glycoprotein in the serum of AIDS and ARC patients. $J$ Acquir Immune Defic Syndr (1988) 1992, 5:251-256.

10. Klasse PJ, Moore JP. Is there enough gp120 in the body fluids of HIV-1-infected individuals to have biologically significant effects? Virology 2004, 323:1-8.

11. Cummins NW, Rizza SA, Badley AD. How much gp120 is there? J Infect Dis. 2010;201:1273-4. author reply 1274-1275.

12. Gilbert PB, Chiu YL, Allen M, Lawrence DN, Chapdu C, Israel H, Holman D, Keefer MC, Wolff M, Frey SE, Network NHVT. Long-term safety analysis of preventive HIV-1 vaccines evaluated in AIDS vaccine evaluation group NIAID-sponsored Phase I and II clinical trials. Vaccine. 2003;21:2933-47. 
13. Santosuosso M, Righi E, Lindstrom V, Leblanc PR, Poznansky MC. HIV-1 envelope protein gp120 is present at high concentrations in secondary lymphoid organs of individuals with chronic HIV-1 infection. J Infect Dis. 2009;200:1050-3.

14. Trujillo JR, Navia BA, Worth J, Lucey DR, McLane MF, Lee TH, Essex M. High levels of anti-HIV-1 envelope antibodies in cerebrospinal fluid as compared to serum from patients with AIDS dementia complex. J Acquir Immune Defic Syndr Hum Retrovirol. 1996;12:19-25.

15. Smith LK, Kuhn TB, Chen J, Bamburg JR. HIV Associated Neurodegenerative Disorders: A New Perspective on the Role of Lipid Rafts in Gp120-Mediated Neurotoxicity. Curr HIV Res. 2018;16:25869.

16. McIntosh RC, Rosselli M, Uddin LQ, Antoni M. Neuropathological sequelae of Human Immunodeficiency Virus and apathy: A review of neuropsychological and neuroimaging studies. Neurosci Biobehav Rev. 2015;55:147-64.

17. Timilsina U, Gaur R. Modulation of apoptosis and viral latency - an axis to be well understood for successful cure of human immunodeficiency virus. J Gen Virol. 2016;97:813-24.

18. Kapadia F, Vlahov D, Donahoe RM, Friedland G. The role of substance abuse in HIV disease progression: reconciling differences from laboratory and epidemiologic investigations. Clin Infect Dis. 2005;41:1027-34.

19. Chilunda V, Calderon TM, Martinez-Aguado P, Berman JW. The impact of substance abuse on HIVmediated neuropathogenesis in the current ART era. Brain Res. 2019;1724:146426.

20. Marquez C, Mitchell SJ, Hare CB, John M, Klausner JD. Methamphetamine use, sexual activity, patient-provider communication, and medication adherence among HIV-infected patients in care, San Francisco 2004-2006. AIDS Care. 2009;21:575-82.

21. Mitchell SJ, Morris SR, Kent CK, Stansell J, Klausner JD. Methamphetamine use and sexual activity among HIV-infected patients in care-San Francisco, 2004. AIDS patient care and STDs 2006, 20:502-510.

22. Gonzales R, Mooney L, Rawson RA. The methamphetamine problem in the United States. Annu Rev Public Health. 2010;31:385-98.

23. Henry BL, Geyer MA, Buell M, Perry W, Young JW, Minassian A. Behavioral effects of chronic methamphetamine treatment in HIV-1 gp120 transgenic mice. Behav Brain Res. 2013;236:210-20.

24. Hoefer MM, Sanchez AB, Maung R, de Rozieres CM, Catalan IC, Dowling CC, Thaney VE, Pina-Crespo J, Zhang D, Roberts AJ, Kaul M. Combination of methamphetamine and HIV-1 gp120 causes distinct long-term alterations of behavior, gene expression, and injury in the central nervous system. Exp Neurol. 2015;263:221-34.

25. Kesby JP, Heaton RK, Young JW, Umlauf A, Woods SP, Letendre SL, Markou A, Grant I, Semenova S. Methamphetamine Exposure Combined with HIV-1 Disease or gp120 Expression: Comparison of Learning and Executive Functions in Humans and Mice. Neuropsychopharmacology. 2015;40:1899909. 
26. Thaney VE, Sanchez AB, Fields JA, Minassian A, Young JW, Maung R, Kaul M. Transgenic mice expressing HIV-1 envelope protein gp120 in the brain as an animal model in neuroAIDS research. $J$ Neurovirol. 2018;24:156-67.

27. Mattson MP, Haughey NJ, Nath A. Cell death in HIV dementia. Cell Death Differ. 2005;12(Suppl 1):893-904.

28. Larsen KE, Fon EA, Hastings TG, Edwards RH, Sulzer D. Methamphetamine-induced degeneration of dopaminergic neurons involves autophagy and upregulation of dopamine synthesis. The Journal of neuroscience: the official journal of the Society for Neuroscience. 2002;22:8951-60.

29. Silverstein PS, Shah A, Weemhoff J, Kumar S, Singh DP, Kumar A. HIV-1 gp120 and drugs of abuse: interactions in the central nervous system. Curr HIV Res. 2012;10:369-83.

30. Chana G, Everall IP, Crews L, Langford D, Adame A, Grant I, Cherner M, Lazzaretto D, Heaton R, Ellis R, Masliah E. Cognitive deficits and degeneration of interneurons in HIV+ methamphetamine users. Neurology. 2006;67:1486-9.

31. Knierim JJ. The hippocampus. Curr Biol. 2015;25:R1116-21.

32. Bird CM, Burgess $N$. The hippocampus and memory: insights from spatial processing. Nat Rev Neurosci. 2008;9:182-94.

33. Thompson PM, Hayashi KM, Simon SL, Geaga JA, Hong MS, Sui Y, Lee JY, Toga AW, Ling W, London ED. Structural abnormalities in the brains of human subjects who use methamphetamine. $J$ Neurosci. 2004;24:6028-36.

34. Castelo JM, Sherman SJ, Courtney MG, Melrose RJ, Stern CE. Altered hippocampal-prefrontal activation in HIV patients during episodic memory encoding. Neurology. 2006;66:1688-95.

35. Kelschenbach J, He H, Kim BH, Borjabad A, Gu CJ, Chao W, Do M, Sharer LR, Zhang H, Arancio O, et al: Efficient Expression of HIV in Immunocompetent Mouse Brain Reveals a Novel Nonneurotoxic
Viral Function in Hippocampal Synaptodendritic Injury and Memory Impairment. mBio 2019, 10.

36. Xiong H, Baskys A, Wojtowicz JM. Brain-derived peptides inhibit synaptic transmission via presynaptic GABAB receptors in CA1 area of rat hippocampal slices. Brain Res. 1996;737:188-94.

37. Morgan SL, Teyler TJ. Electrical stimuli patterned after the theta-rhythm induce multiple forms of LTP. J Neurophysiol. 2001;86:1289-96.

38. Liu J, Xu E, Tu G, Liu H, Luo J, Xiong H. Methamphetamine potentiates HIV-1gp120-induced microglial neurotoxic activity by enhancing microglial outward $\mathrm{K}(+)$ current. Molecular and cellular neurosciences 2017, 82:167-175.

39. Xu E, Liu J, Liu H, Wang X, Xiong H. Inflammasome Activation by Methamphetamine Potentiates Lipopolysaccharide Stimulation of IL-1 beta Production in Microglia. J Neuroimmune Pharmacol. 2018;13:237-53.

40. Markram H, Gupta A, Uziel A, Wang Y, Tsodyks M. Information processing with frequency-dependent synaptic connections. Neurobiol Learn Mem. 1998;70:101-12. 
41. Garcia-Calvo M, Peterson EP, Leiting B, Ruel R, Nicholson DW, Thornberry NA. Inhibition of human caspases by peptide-based and macromolecular inhibitors. J Biol Chem. 1998;273:32608-13.

42. Goldstein RZ, Volkow ND. Dysfunction of the prefrontal cortex in addiction: neuroimaging findings and clinical implications. Nat Rev Neurosci. 2011;12:652-69.

43. Takeuchi T, Duszkiewicz AJ, Morris RG. The synaptic plasticity and memory hypothesis: encoding, storage and persistence. Philos Trans R Soc Lond B Biol Sci. 2014;369:20130288.

44. Neves G, Cooke SF, Bliss TV. Synaptic plasticity, memory and the hippocampus: a neural network approach to causality. Nat Rev Neurosci. 2008;9:65-75.

45. Dudek SM, Bear MF. Homosynaptic long-term depression in area CA1 of hippocampus and effects of N-methyl-D-aspartate receptor blockade. Proc Natl Acad Sci U S A. 1992;89:4363-7.

46. Abraham WC, Bliss TV, Goddard GV. Heterosynaptic changes accompany long-term but not shortterm potentiation of the perforant path in the anaesthetized rat. J Physiol. 1985;363:335-49.

47. Brace HM, Jefferys JG, Mellanby J. Long-term changes in hippocampal physiology and learning ability of rats after intrahippocampal tetanus toxin. J Physiol. 1985;368:343-57.

48. Abraham WC, Jones OD, Glanzman DL. Is plasticity of synapses the mechanism of long-term memory storage? NPJ Sci Learn. 2019;4:9.

49. Kallianpur KJ, Shikuma C, Kirk GR, Shiramizu B, Valcour V, Chow D, Souza S, Nakamoto B, Sailasuta N. Peripheral blood HIV DNA is associated with atrophy of cerebellar and subcortical gray matter. Neurology. 2013;80:1792-9.

50. Carey CL, Woods SP, Rippeth JD, Gonzalez R, Heaton RK, Grant I. Additive deleterious effects of methamphetamine dependence and immunosuppression on neuropsychological functioning in HIV infection. AIDS Behav. 2006;10:185-90.

51. Jana A, Pahan K. Human immunodeficiency virus type 1 gp120 induces apoptosis in human primary neurons through redox-regulated activation of neutral sphingomyelinase. J Neurosci. 2004;24:953140.

52. Price TO, Ercal N, Nakaoke R, Banks WA. HIV-1 viral proteins gp120 and Tat induce oxidative stress in brain endothelial cells. Brain research. 2005;1045:57-63.

53. Kanmogne GD, Schall K, Leibhart J, Knipe B, Gendelman HE, Persidsky Y. HIV-1 gp120 compromises blood-brain barrier integrity and enhances monocyte migration across blood-brain barrier: implication for viral neuropathogenesis. J Cereb Blood Flow Metab. 2007;27:123-34.

54. Louboutin JP, Reyes BA, Agrawal L, Van Bockstaele EJ, Strayer DS. HIV-1 gp120-induced neuroinflammation: relationship to neuron loss and protection by rSV40-delivered antioxidant enzymes. Exp Neurol. 2010;221:231-45.

55. Loftis JM, Janowsky A. Neuroimmune basis of methamphetamine toxicity. International review of neurobiology. 2014;118:165-97.

56. Shaerzadeh F, Streit WJ, Heysieattalab S, Khoshbouei H. Methamphetamine neurotoxicity, microglia, and neuroinflammation. J Neuroinflammation. 2018;15:341. 
57. Dong J, Xiong H. Human immunodeficiency virus type 1 gp120 inhibits long-term potentiation via chemokine receptor CXCR4 in rat hippocampal slices. J Neurosci Res. 2006;83:489-96.

58. Shen LL, Jiang ML, Liu SS, Cai MC, Hong ZQ, Lin LQ, Xing YY, Chen GL, Pan R, Yang LJ, et al. Curcumin improves synaptic plasticity impairment induced by HIV-1gp120 V3 loop. Neural Regen Res. 2015;10:925-31.

59. Sanchez-Alavez M, Criado J, Gomez-Chavarin M, Jimenez-Anguiano A, Navarro L, Diaz-Ruiz O, Galicia O, Sanchez-Narvaez F, Murillo-Rodriguez E, Henriksen SJ, et al. HIV- and FIV-derived gp120 alter spatial memory, LTP, and sleep in rats. Neurobiol Dis. 2000;7:384-94.

60. Krucker T, Toggas SM, Mucke L, Siggins GR. Transgenic mice with cerebral expression of human immunodeficiency virus type-1 coat protein gp120 show divergent changes in short- and long-term potentiation in CA1 hippocampus. Neuroscience. 1998;83:691-700.

61. Swant J, Chirwa S, Stanwood G, Khoshbouei H. Methamphetamine reduces LTP and increases baseline synaptic transmission in the CA1 region of mouse hippocampus. PLoS One. 2010;5:e11382.

62. Onaivi ES, Ali SF, Chirwa SS, Zwiller J, Thiriet N, Akinshola BE, Ishiguro H. Ibogaine signals addiction genes and methamphetamine alteration of long-term potentiation. Ann N Y Acad Sci. 2002;965:2846.

63. Hori N, Kadota MT, Watanabe M, Ito Y, Akaike N, Carpenter DO. Neurotoxic effects of methamphetamine on rat hippocampus pyramidal neurons. Cell Mol Neurobiol. 2010;30:849-56.

64. Shahidi S, Komaki A, Sadeghian R, AsI SS. Different doses of methamphetamine alter long-term potentiation, level of BDNF and neuronal apoptosis in the hippocampus of reinstated rats. J Physiol Sci. 2019;69:409-19.

65. Ishikawa A, Kadota T, Kadota K, Matsumura H, Nakamura S. Essential role of D1 but not D2 receptors in methamphetamine-induced impairment of long-term potentiation in hippocampal-prefrontal cortex pathway. Eur J Neurosci. 2005;22:1713-9.

66. Huang X, Chen YY, Shen Y, Cao X, Li A, Liu Q, Li Z, Zhang LB, Dai W, Tan T, et al. Methamphetamine abuse impairs motor cortical plasticity and function. Mol Psychiatry. 2017;22:1274-81.

67. Baptista S, Lourenco J, Milhazes N, Borges F, Silva AP, Bacci A. Long-Term Treatment with Low Doses of Methamphetamine Promotes Neuronal Differentiation and Strengthens Long-Term
Potentiation of Glutamatergic Synapses onto Dentate Granule Neurons. eNeuro 2016, 3.

68. Yun SH, Mook-Jung I, Jung MW. Variation in effective stimulus patterns for induction of long-term potentiation across different layers of rat entorhinal cortex. J Neurosci. 2002;22:RC214.

69. Hernandez RV, Navarro MM, Rodriguez WA, Martinez JL Jr, LeBaron RG. Differences in the magnitude of long-term potentiation produced by theta burst and high frequency stimulation protocols matched in stimulus number. Brain Res Brain Res Protoc. 2005;15:6-13.

70. Buzsaki G. Theta oscillations in the hippocampus. Neuron. 2002;33:325-40.

71. O'Keefe J, Recce ML. Phase relationship between hippocampal place units and the EEG theta rhythm. Hippocampus. 1993;3:317-30. 
72. Stepan J, Dine J, Fenzl T, Polta SA, von Wolff G, Wotjak CT, Eder M. Entorhinal theta-frequency input to the dentate gyrus trisynaptically evokes hippocampal CA1 LTP. Front Neural Circuits. 2012;6:64.

73. Rodrigues NC, Silva-Cruz A, Caulino-Rocha A, Bento-Oliveira A, Alexandre Ribeiro J, Cunha-Reis D. Hippocampal CA1 theta burst-induced LTP from weaning to adulthood: Cellular and molecular mechanisms in young male rats revisited. Eur J Neurosci. 2021;54:5272-92.

74. Larson J, Munkácsy E. Theta-burst LTP. Brain Res. 2015;1621:38-50.

75. Fall CP, Rinzel J. An intracellular Ca2+ subsystem as a biologically plausible source of intrinsic conditional bistability in a network model of working memory. J Comput Neurosci. 2006;20:97-107.

76. Zhu Y, Wang R, Wang Y. A Comparative Study of the Impact of Theta-Burst and High-Frequency Stimulation on Memory Performance. Front Hum Neurosci. 2016;10:19.

77. Landfield PW, McGaugh JL, Tusa RJ. Theta rhythm: a temporal correlate of memory storage processes in the rat. Science. 1972;175:87-9.

78. Shah A, Kumar S, Simon SD, Singh DP, Kumar A. HIV gp120- and methamphetamine-mediated oxidative stress induces astrocyte apoptosis via cytochrome P450 2E1. Cell death disease. 2013;4:e850.

79. Teodorof-Diedrich C, Spector SA. Human Immunodeficiency Virus Type 1 and MethamphetamineMediated Mitochondrial Damage and Neuronal Degeneration in Human Neurons. J Viro/ 2020, 94.

80. Mahajan SD, Aalinkeel R, Sykes DE, Reynolds JL, Bindukumar B, Adal A, Qi M, Toh J, Xu G, Prasad PN, Schwartz SA. Methamphetamine alters blood brain barrier permeability via the modulation of tight junction expression: Implication for HIV-1 neuropathogenesis in the context of drug abuse. Brain Res. 2008;1203:133-48.

81. Ohene-Nyako M, Persons AL, Napier TC. Hippocampal blood-brain barrier of methamphetamine selfadministering HIV-1 transgenic rats. Eur J Neurosci. 2021;53:416-29.

82. Parnet P, Amindari S, Wu C, Brunke-Reese D, Goujon E, Weyhenmeyer JA, Dantzer R, Kelley KW. Expression of type I and type II interleukin-1 receptors in mouse brain. Brain Res Mol Brain Res. 1994;27:63-70.

83. Hoshino K, Hayakawa M, Morimoto Y. Minocycline Prevents the Impairment of Hippocampal LongTerm Potentiation in the Septic Mouse. Shock. 2017;48:209-14.

84. Hoshino K, Uchinami Y, Uchida Y, Saito H, Morimoto Y. Interleukin-1beta Modulates Synaptic Transmission and Synaptic Plasticity During the Acute Phase of Sepsis in the SenescenceAccelerated Mouse Hippocampus. Front Aging Neurosci. 2021;13:637703.

85. Katsuki H, Nakai S, Hirai Y, Akaji K, Kiso Y, Satoh M. Interleukin-1 beta inhibits long-term potentiation in the CA3 region of mouse hippocampal slices. Eur J Pharmacol. 1990;181:323-6.

86. Bellinger FP, Madamba S, Siggins GR. Interleukin 1 beta inhibits synaptic strength and long-term potentiation in the rat CA1 hippocampus. Brain Res. 1993;628:227-34.

87. Schneider H, Pitossi F, Balschun D, Wagner A, del Rey A, Besedovsky HO. A neuromodulatory role of interleukin-1beta in the hippocampus. Proc Natl Acad Sci U S A. 1998;95:7778-83. 
88. Hoshino K, Hasegawa K, Kamiya H, Morimoto Y. Synapse-specific effects of IL-1beta on long-term potentiation in the mouse hippocampus. Biomed Res. 2017;38:183-8.

89. Melega WP, Cho AK, Harvey D, Lacan G. Methamphetamine blood concentrations in human abusers: application to pharmacokinetic modeling. Synapse. 2007;61:216-20.

90. Riviere GJ, Gentry WB, Owens SM. Disposition of methamphetamine and its metabolite amphetamine in brain and other tissues in rats after intravenous administration. J Pharmacol Exp Ther. 2000;292:1042-7.

\section{Figures}


A
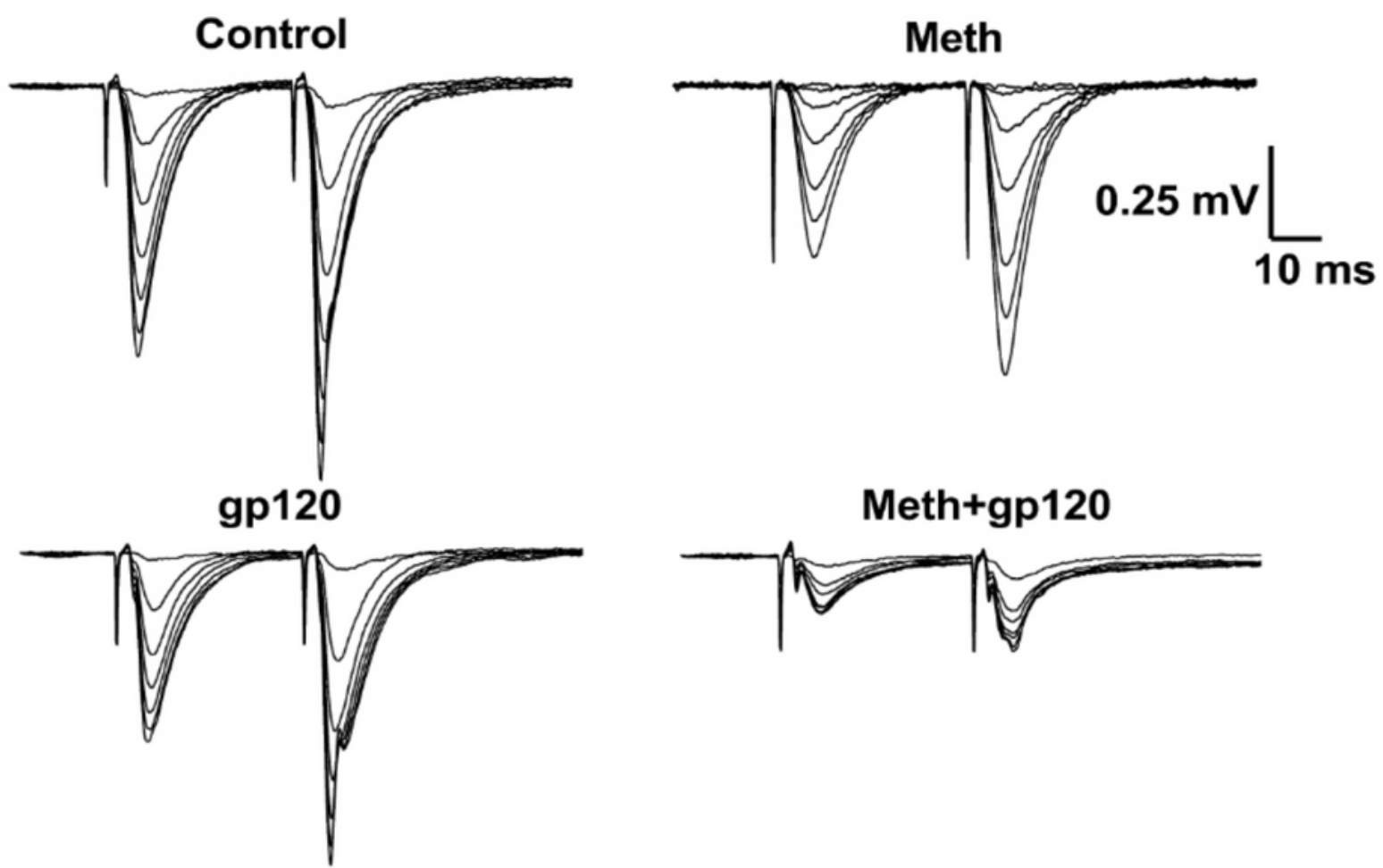

B

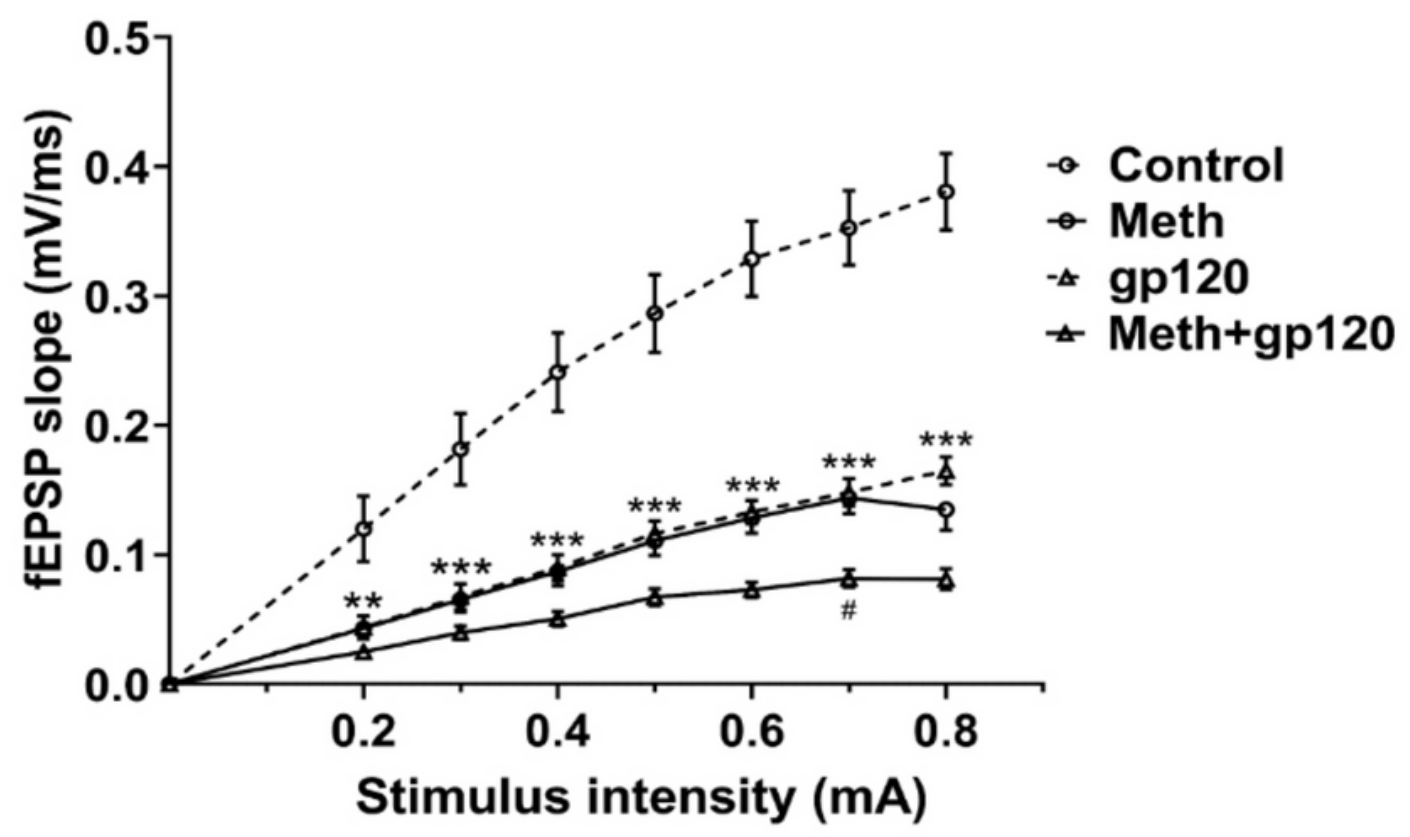

Figure 1

Inhibitory effects of Meth and HIV-1 gp120 on synaptic transmission in the CA1 region of rat hippocampal slices. (A) Representative input-output (traces of superimposed fEPSPs, evoked by twinpulse stimuli (10 $\mu \mathrm{s}$ in duration, $40 \mathrm{~ms}$ inter-pulse interval) at various intensities ranging from 0.2 to 0.8 $\mathrm{mA}$ with an increment of $0.1 \mathrm{~mA}$, were recorded from hippocampal slices with different treatments as indicated. Each trace shown was an average of consecutive 3 evoked fEPSPs. (B) Averaged input-output 
curves generated by plotting fEPSP initial slope of the first pulse against the stimulus intensities ranging from 0.2 to $0.8 \mathrm{~mA}$ as shown in A. Note that Meth and gp120 each alone significantly decreased the slopes of fEPSP evoked by stimuli with all different intensities. Combined application further deceased the fEPSP slope at stimuli intensities of $0.7 \mathrm{~mA}$ and $0.8 \mathrm{~mA}$ compared to Meth and gp120 each alone. Data represent mean \pm SEM and are analyzed using two-way ANOVA followed by Tukey's post hoc test. **P $<0.01$, *** $\mathrm{P}<0.001$ vs. control, $\# \mathrm{P}<0.05$ vs. Meth.

A
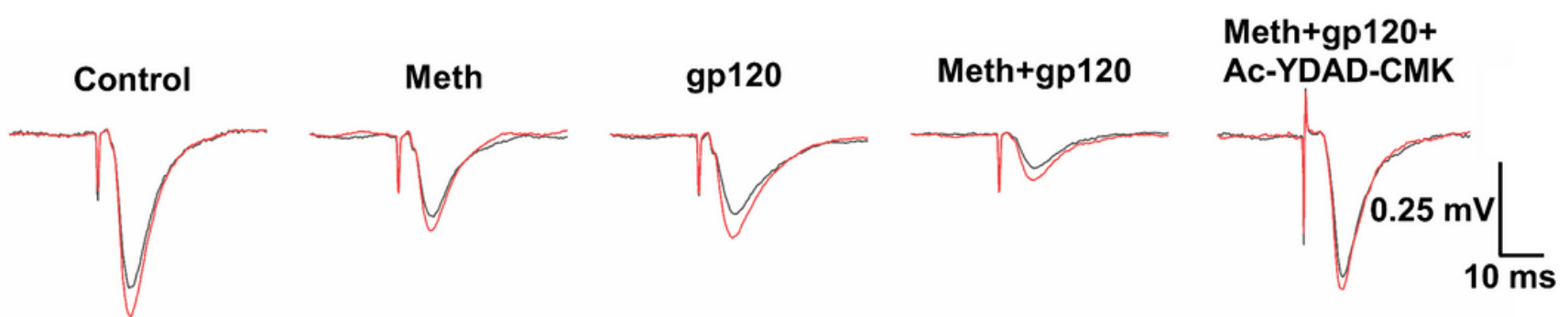

B

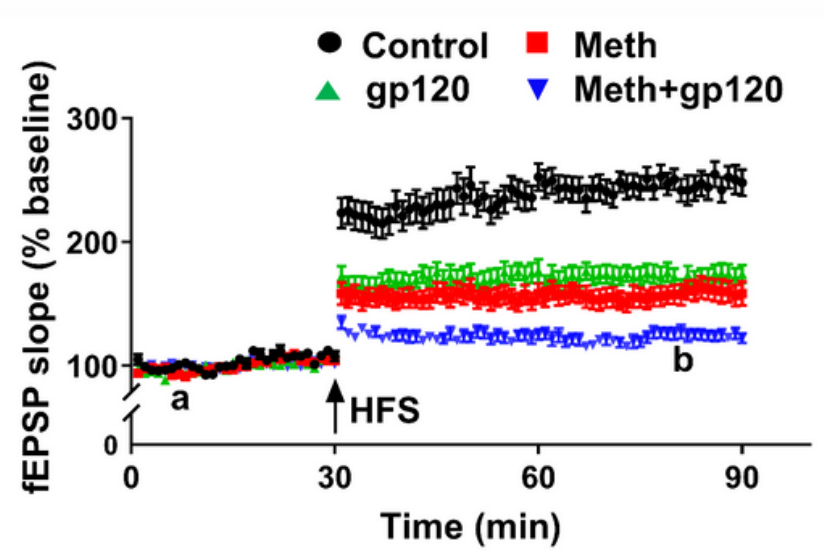

C

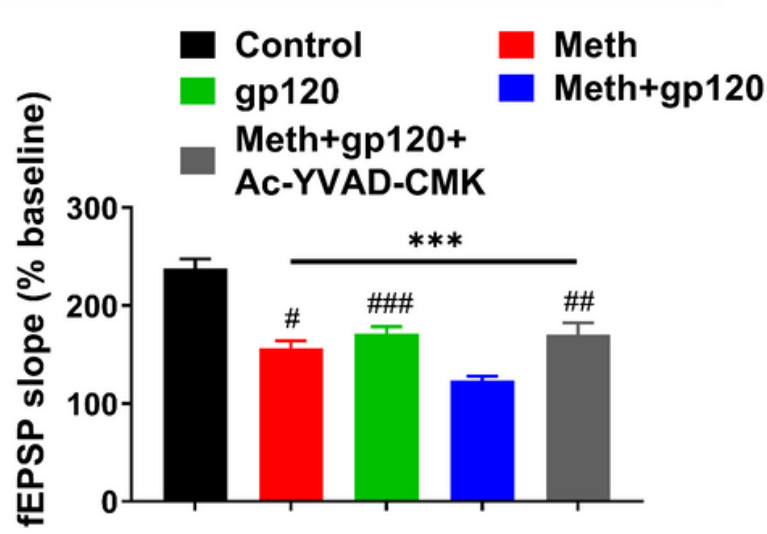

D

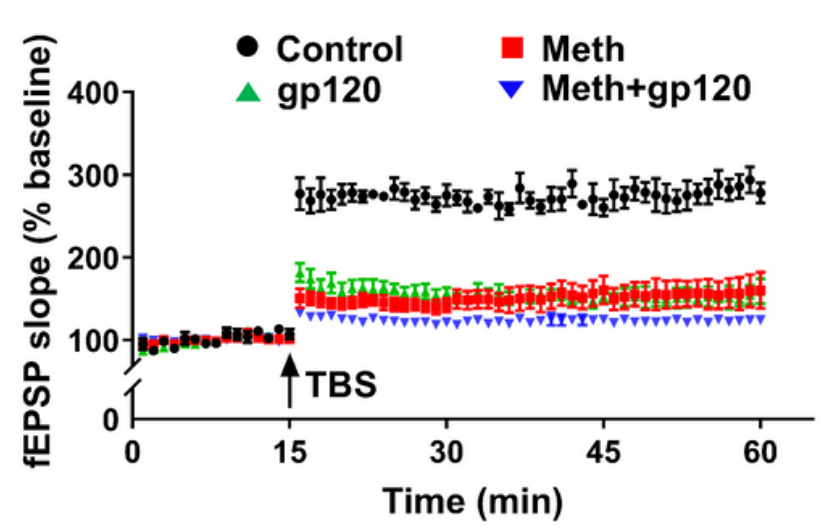

E

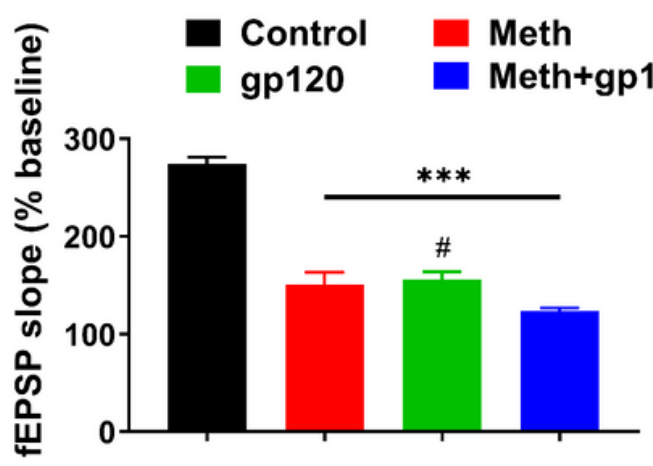

Figure 2

Meth augments HIV-1 gp120 inhibition of long-term potentiation (LTP) induced by either high-frequency stimulation (HFS) or theta-burst stimulation (TBS). Panel A shows the representative fEPSPs taken at the time points of a (base line, in black) and b (50 min after HFS, in red) at different experimental condition as shown in Panel B. Panels B and D illustrate the time courses and average magnitudes of LTP recorded in the Schaffer-collateral to CA1 synapses induced by HFS (B) or TBS (D) under different experimental 
conditions as indicated. Note that the time course and average magnitudes of LTP for Meth+gp120+AcYDAD-CMK group were not shown in Panel $B$ due to over-crowed curves and overlap. Panels $C$ and $E$ are summarized bar graphs for Panels $B$ and D respectively, showing that Meth and gp120 each alone significantly reduced the magnitude (an average number of LTP period) of LTP induced by HFS (C) and TBS (E). However, Meth augmented gp120 reduction of LTP magnitude when tested in combination. Addition of caspase 1 inhibitor Ac-YDAD-CMK blocked Meth-associated augmentation of gp120 reduction on LTP magnitude, suggesting an involvement of inflammasome in such an augmentation. Sample size (n) in groups of HFS and TBS (in brackets): Control 23 (5), Meth 22 (6), gp120 20 (6), Meth+gp120 20 (7), Meth+gp120+Ac-YVAD-CMK 10. Data represent the mean \pm SEM and are analyzed

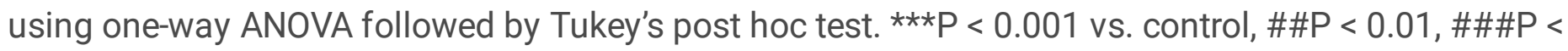
0.001 vs. Meth+gp120.
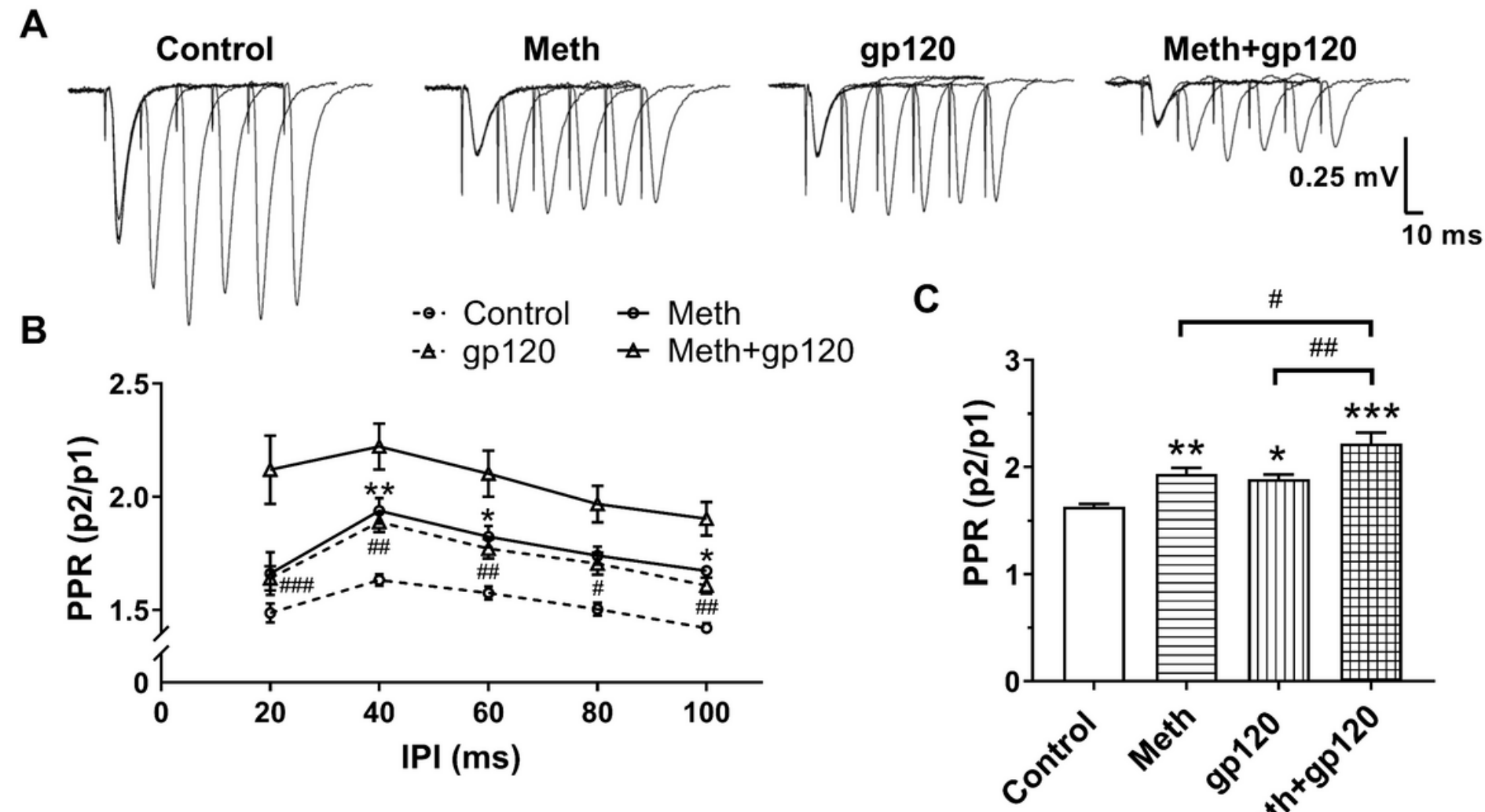

\section{Figure 3}

Meth- and gp120-induced alteration of paired-pulse facilitation. Effects of Meth and gp120 on pairedpulse facilitation (PPF) were tested at the Schaffer-collateral to CA1 synapses in the hippocampal slices. fEPSPs were elicited by paired-pulse stimulation ( $10 \mu \mathrm{s}$ in duration, $200 \mu \mathrm{A}$ in intensity) with varying interpulse interval (IPI) from 20 to $100 \mathrm{~ms}$ with an increment of $20 \mathrm{~ms}$. The paired-pulse facilitation ratio (PPR) was calculated by dividing the first fEPSP peak amplitude from the second fEPSP peak amplitude. (A) Representative superimposed traces of fEPSPs recorded at indicated experimental conditions in response to paired-pulse stimulations with different IPIs. (B) Line graph plots the magnitudes of PPRs 
against IPIs. Note a decrease in second responses as IPI increase starting from $40 \mathrm{~ms}$. (C) Bar graph shows the PPRs at $40 \mathrm{~ms}$ IPI in different experimental conditions. Note Meth and g120 each alone increased PPR and further significant increase in PPR was observed when Meth and gp120 was tested in combination, illustrating a presynaptic site of action. Data represent the mean \pm SEM with $n=20$ in each group and are analyzed using two-way ANOVA (B) and one-way ANOVA followed by Tukey's post hoc test

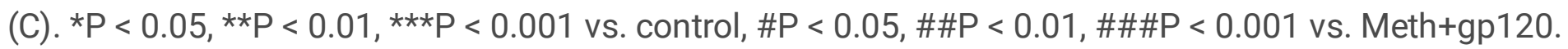

A
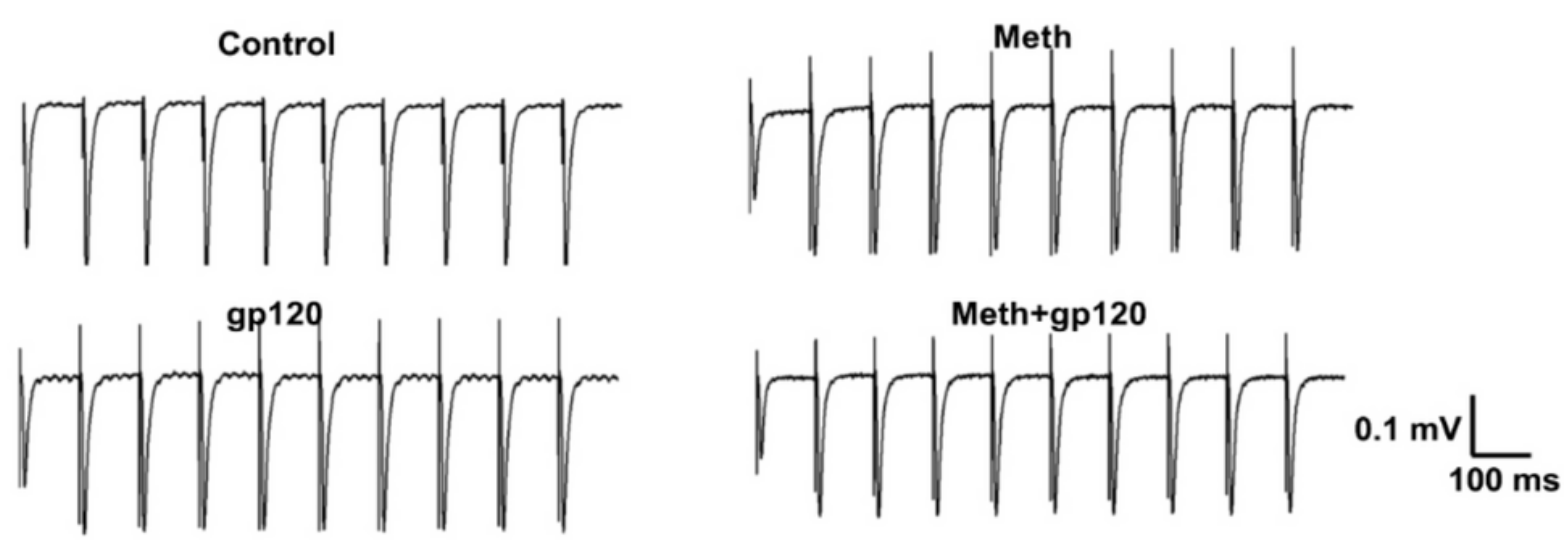

B

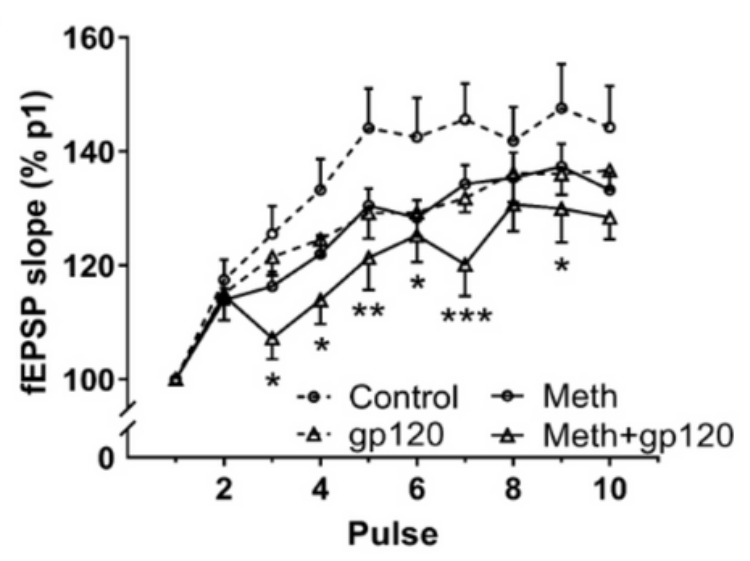

C

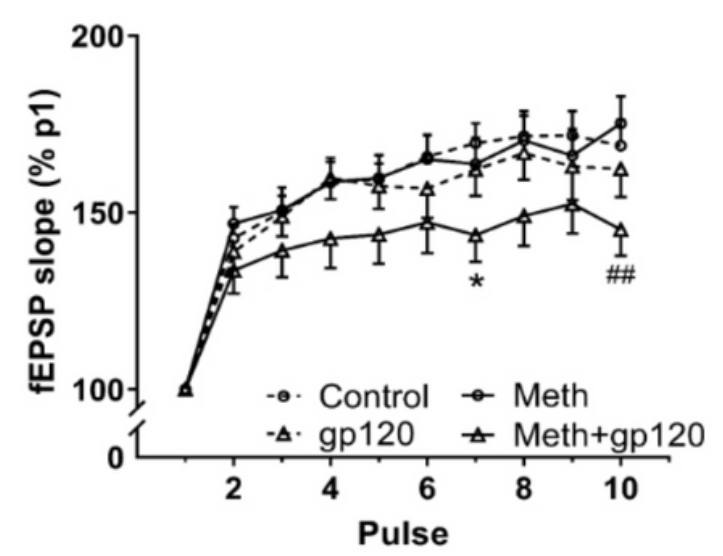

D

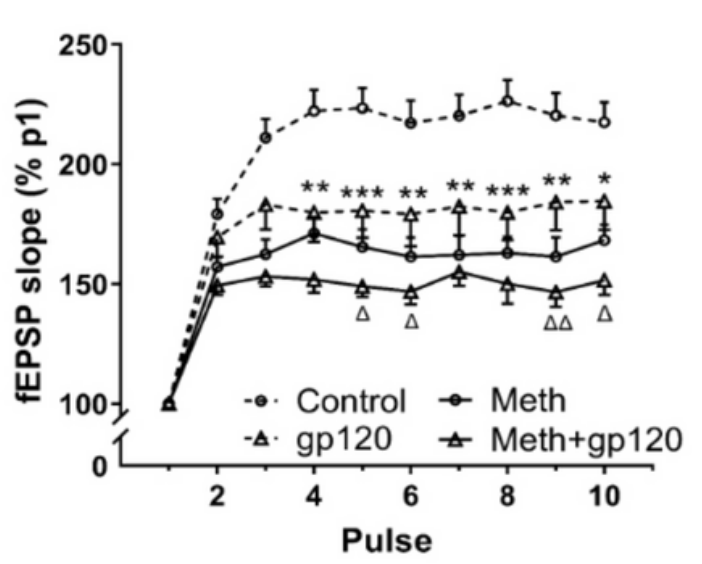

E

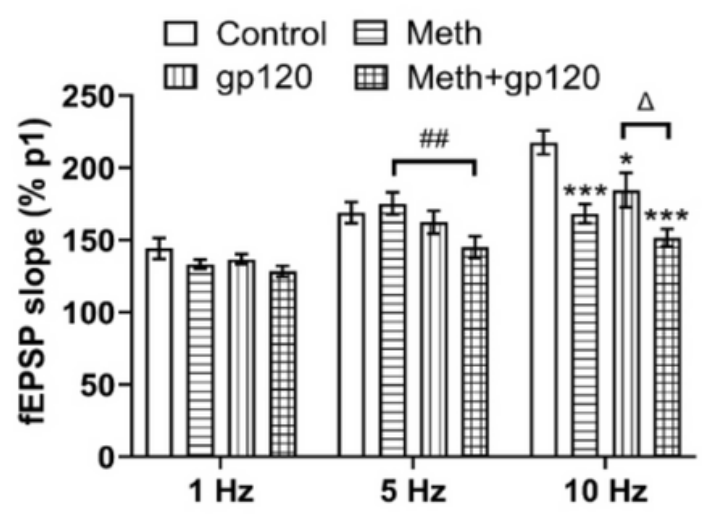

Figure 4 
Meth- and gp120-associated alteration of frequency facilitation of synaptic responses. The influence of Meth and gp12 on frequency facilitation (FF) of synaptic responses were examined in the CA1 region of hippocampal slices. Comparisons were made among frequency responses generated in response to a 10pulse stimulation ( $10 \mu \mathrm{s}$ in duration, $0.2 \mathrm{~mA}$ in intensity, ) at $1.0 \mathrm{~Hz}, 5.0 \mathrm{~Hz}$ and $10.0 \mathrm{~Hz}$. The degree of potentiation was expressed as the percentage of initial slopes of the 2-10th pulses over the first one (regarding the first pulse as 100\%). (A) Exemplary FF recordings at the frequency of $10 \mathrm{~Hz}$ from four experimental conditions. Each trace shown was an average of consecutive 3 evoked fEPSPs. (B), (C) and (D) are line graphs exhibiting average normalized fEPSP slopes at the corresponding frequency of $1 \mathrm{~Hz}, 5$ $\mathrm{Hz}$, and $10 \mathrm{~Hz}$. Note significant reductions of FF in some pulses in Meth+gp120 group ( $n=18)$ compared to control $(n=20)$. (E) Summary data of tenth fEPSP slopes obtained at four distinct experimental conditions with different stimulation frequencies. Note that at $10 \mathrm{~Hz}$, Meth $(n=20)$ and gp120 $(n=20)$, applied alone or in combination, decreased FF dramatically when compared with gp120 alone, demonstrating Meth and gp120 impairment of FF in the hippocampus. Data represent the mean \pm SEM and are analyzed using two-way ANOVA followed by Tukey's post hoc test. ${ }^{*} P<0.05,{ }^{*} \mathrm{P}<0.01$, ${ }^{* \star *} \mathrm{P}<$ 0.001 vs. control, \#\#P $<0.01$ vs. Meth, $\Delta \mathrm{P}<0.05, \Delta \Delta \mathrm{P}<0.01$ vs. gp120. 


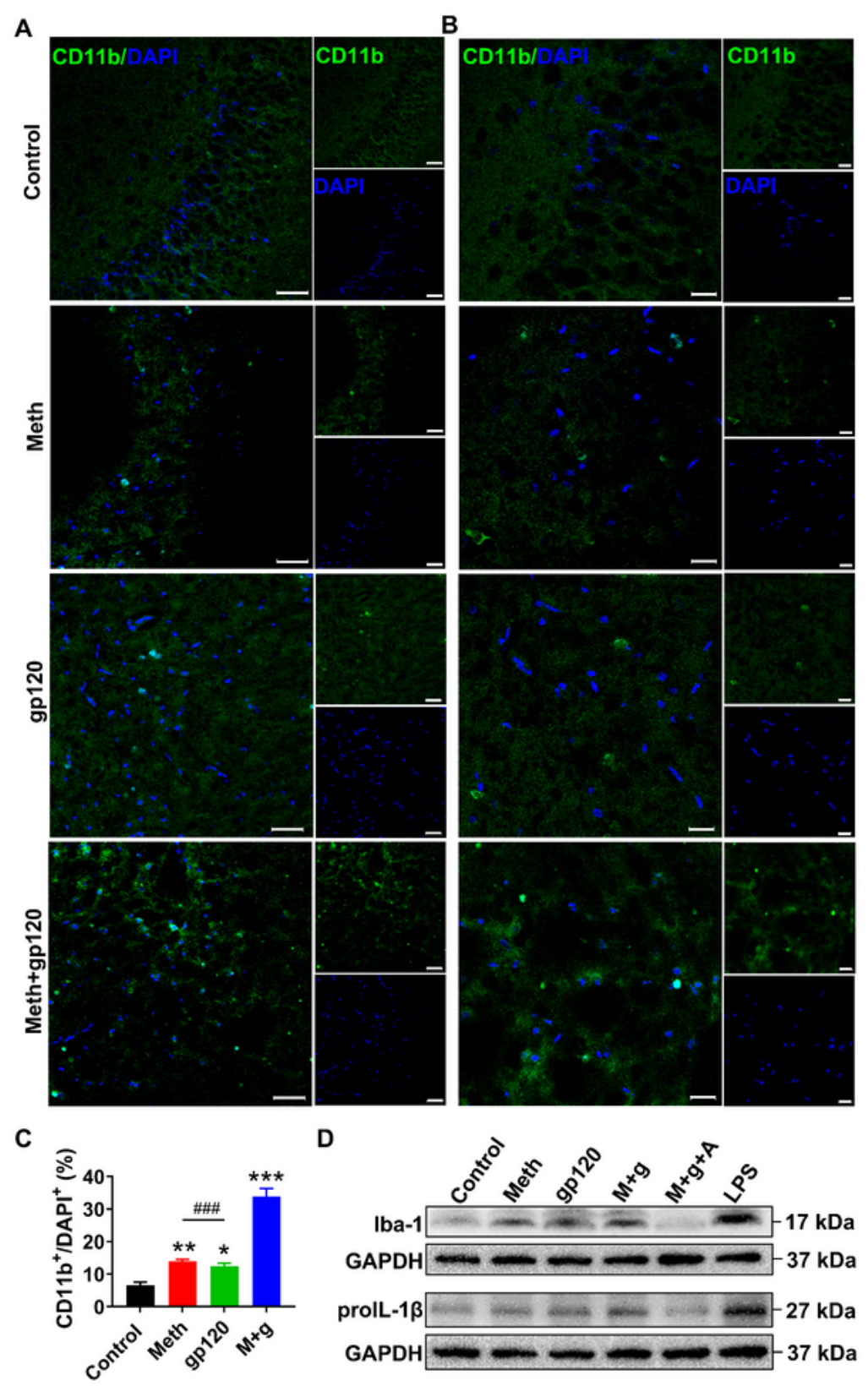

$\mathrm{E}$

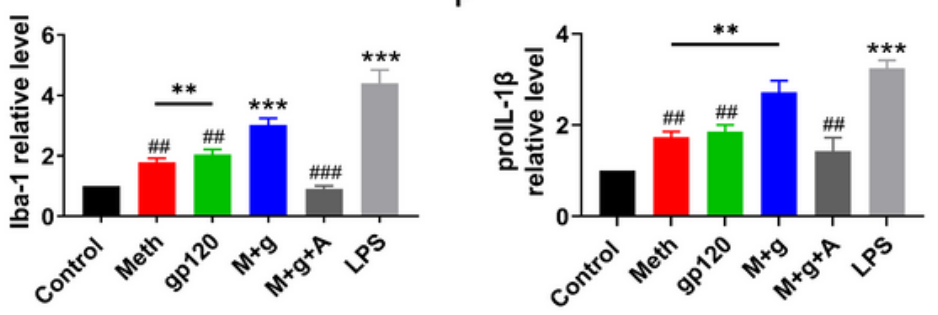

Figure 5

Meth- and gp120-inducbed microglial activation in hippocampal slices. (A) Immunostaining displays expression of $\mathrm{CD} 11 \mathrm{~b}$, a marker of microglial activation, in the rat hippocampal slices in experimental groups as indicated. The right two images (vertical) in each group were the images of CD11b staining (green, upper) and DAPI staining (blue, lower). The left image (larger in size) in each group was an enlarged photo with CD11b-staining image and DAPI-staining image superimposed. Images were 
visualized by fluorescent microscopy at $\times 20$ original magnification. Scale bar equals $50 \mu \mathrm{m}$. (B) Representative images, from each group shown in (A), were visualized by fluorescent microscopy at $x 40$ original magnification. Scale bar equals $20 \mu \mathrm{m}$. (C) Bar graph exhibiting quantification of CD11b+/DAPI+ cells showed that microglial activation was significantly potentiated by Meth and gp120 applied in combination. $\mathrm{N}=10$ in each group (4 independent experiments). (D) Exemplary bands of western blot analysis illustrated the expression levels of Iba-1 and prolL-1 $1 \beta$. (E-F) Bar graphs showing band densitometry data in ( $\mathrm{n}=8$ in each group, 4 independent experiments). Application of Meth and gp120 each alone significantly $(p<0.01)$ increased the expression levels of Iba- 1 and prolL-1 $\beta$. However, a more significant increase $(p<0.001)$ was observed when applied in combination. Note that pretreatment of slices with caspase-1 inhibitor Ac-YVAD-CMK $(10 \mu \mathrm{M})$ for $1 \mathrm{hr}$ blocked the combined effect of Meth and gp120. LPS was tested as a positive control. Data are presented as mean \pm SEM and analyzed by oneway ANOVA followed by Turkey's post hoc test. ${ }^{\star} p<0.05$, ${ }^{\star \star} p<0.01$, ${ }^{\star \star \star} p<0.001$ vs. control, \#\#p<0.01, $\# \# \#<0.001$ vs. Meth+gp120. 
A
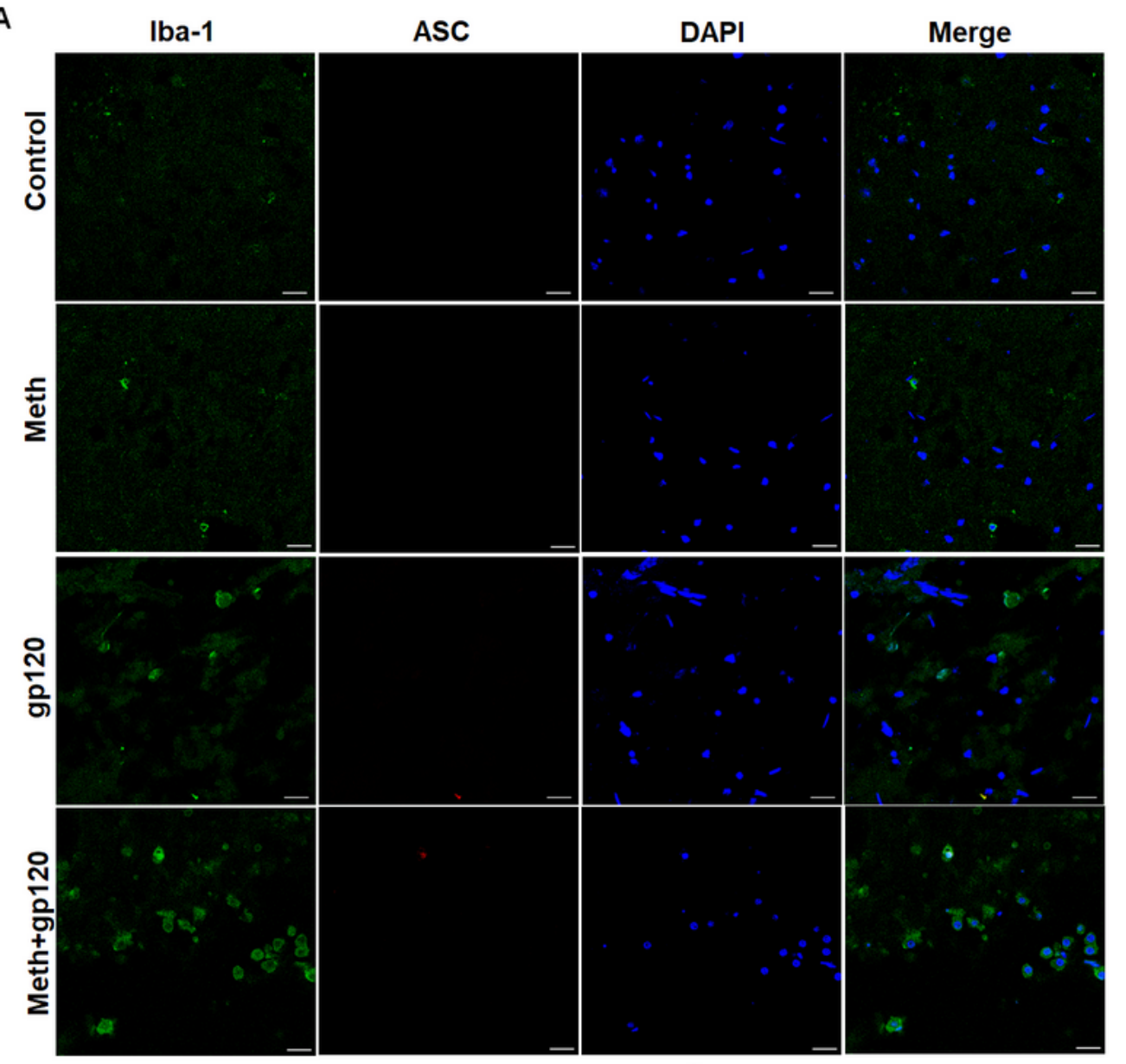

B
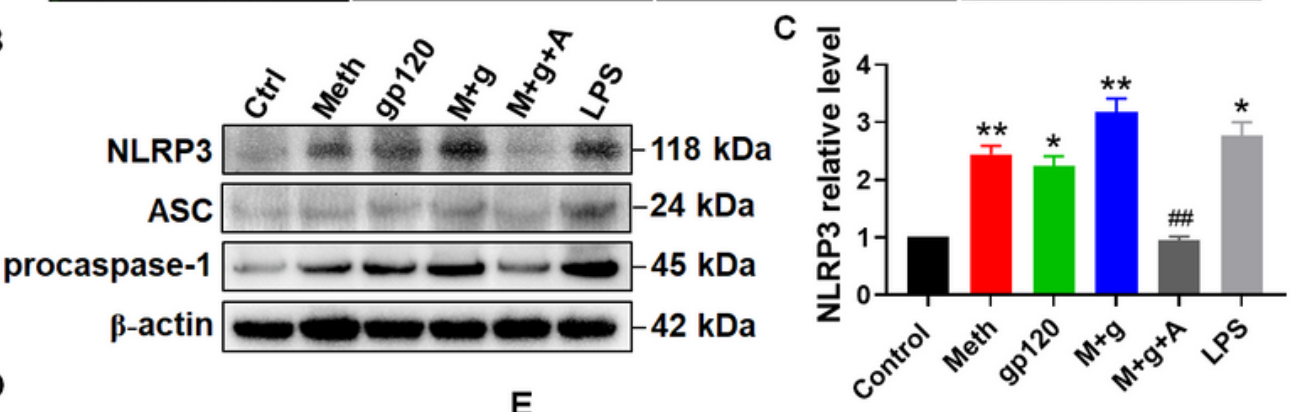

D

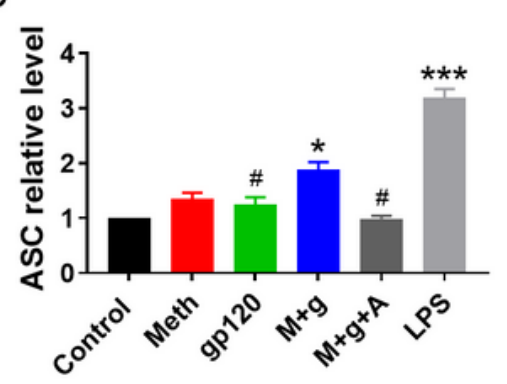

E

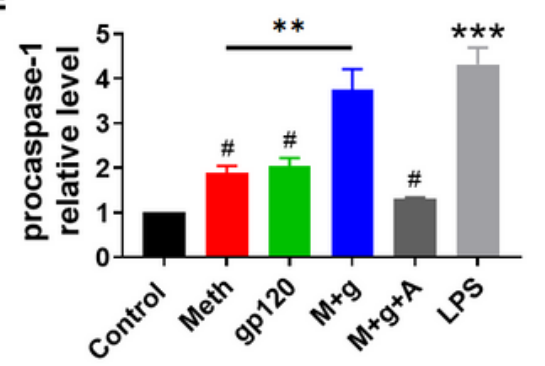

Figure 6

Involvement of NLRP3 inflammasome in synergic microglial activation by Meth and gp120. (A) Representative immunofluorescence images taken from hippocampal slices in four groups with different treatments as indicated. ASC/Iba-1/DAPI triple positive cells were spotted in slices treated with Meth and gp120 in combination, but not in slices treated with each alone. Scale bar equals $20 \mu \mathrm{m}$. (B) Exemplary band of western blot analysis exhibited the expression levels of NLRP3, ASC and procaspase-1. (C-E) 
Band densitometry data from 4 independent experiments, normalized with the controls, showed Meth and gp120 in combination upregulated the expression levels of ASC ( $n=5$, compared to gp120 alone) and procaspase-1 ( $\mathrm{n}=8$, compared to Meth and gp120 each alone). Pretreatment of slices with caspase-1 inhibitor Ac-YVAD-CMK $(10 \mu \mathrm{M})$ for $1 \mathrm{hr}$ significantly blocked the up-regulatory effects of Meth and gp120 on inflammasome complex expression. LPS was used as a positive control. Data are presented as mean \pm SEM and analyzed using one-way ANOVA followed by Tukey's post hoc test. ${ }^{*} P<0.05$, ${ }^{\star *} \mathrm{P}<0.01$, $* \star * P$ $<0.001$ vs. control, \#P < 0.05, \#\#P $<0.01$ vs. Meth+gp120. 